\title{
Tax Contribution and Income Gap between Urban and Rural Areas in China
}

\author{
Yichao Yu \\ School of Economics, Jinan University, Guangzhou, China \\ Email: 576505073@qq.com
}

Received 20 October 2015; accepted 14 November 2015; published 17 November 2015

Copyright (C) 2015 by author and Scientific Research Publishing Inc.

This work is licensed under the Creative Commons Attribution International License (CC BY). http://creativecommons.org/licenses/by/4.0/

cC) (i) Open Access

\begin{abstract}
This article analyses the relationship of tax contribution and income gap between urban and rural areas. First of all, we comb their relationship from theoretical knowledge. Secondly, we use 20002014 panel data of 29 provinces and cities in our country (except Tibet) to establish the fixed effects model for analysis. Results show that the improvement of tax contribution will expand the income gap between urban and rural areas. This is due to that turnover tax contribution is the most important part in the tax contribution. From the structural analysis, improvement of turnover tax and income tax contribution are not conducive to narrow the income gap between urban and rural areas. The improvement of property tax contribution is conducive to narrow the income gap between urban and rural areas. Finally, from the empirical results, we can give the policy suggestion of structural tax cuts and others.
\end{abstract}

\section{Keywords}

Income Gap between Urban and Rural Areas, Tax Contribution, Fixed Effects Model, Structural Tax Cuts

\section{Introduction}

Since 1978, our country's economy maintained a high speed development, the per capita GDP rose from 381 yuan in 1978 to 46,531 yuan in 2014, growth in 122 times. At the same time, income of urban and rural residents had greatly improved. Urban per capita disposable income in 1978 was 343 yuan. The per capita disposable income reached 28,844 yuan in 2014. Rural resident per capita net income of 1978 was 134 yuan, and it reached 10489 yuan in 2014. However, with the rapid growth of economy and increase of residents' income, the problem of income distribution was increasingly prominent in our country, specially the income gap between urban and rural areas. Urban and rural income ratio was 2.75 times in 2014. The income gap between urban and 
rural areas had become one of the important problems that we need to resolve now. Tax is an important means of government to adjust the income distribution. By studying the relationship of tax and the income gap between urban and rural areas in China, it helps us to put forward policy suggestions with narrowing the income gap between urban and rural areas.

\section{Literature Review}

The earliest domestic scholars studied Chinese income distribution problem in the late 1980s, such as Zongsheng Chen, Wang-Dao Chen. They analyzed income gap between urban and rural areas in China from different angle, such as present situation, evolution characteristic and reason of income gap. To the 90s of 20th century, scholars began to study the relationship between taxation and income distribution, such as Guoqing Wang (1995), Lu Renfa (1996), Xujian Guo (1998) and others. In recent years, tax refund and weakening of the function of the tax fair had become the focus of attention. Domestic literature could be divided into 3 categories:

The first kind is about the position of the relationship between the tax revenue and the income distribution. Tax was an important tool to adjust the income distribution. We could use the progressive tax, tax incentives, tax cuts and other ways to make a fairer income distribution [1]. Pei-Yong Gao thought tax was an important means for government to adjust the income gap between residents. The government should combine tax adjustment work and the construction of the tax system, so that tax system could really play a role in adjusting income gap between residents [2]. In order to improve our tax system and realize the function of adjusting income gap, we need to find the cause of the income gap. If it is not reasonable in the tax system, we need to reform and pay more attention to system construction [3].

The second kind is about empirical research on taxation and income distribution. Hua Liu used data of the world bank to analyze the relationship between turnover tax and income gap. Results show that turnover tax was not conducive to narrow the income gap [4]. Zi Yin Shi used intermediate progressive index to analyze income redistribution effect of personal income tax, the average tax rate played a key role on income redistribution effect [5].

The third kind is about tax refund and weakening of the function of tax fair. Existing research literature had confirmed that tax adjustment function of the income gap was very weak in China, and there was a phenomenon of "reverse adjustment". Xi-Min Yue used the cash flow statement and household survey data to measure tax burden level of each family. Results show that Chinese tax structure was regressive. Compared with urban areas, tax burden of rural areas were more regressive [6]. Ying Wan studied distribution of turnover tax burden. Turnover tax was regressive. Value added tax and business tax was regressive, but consumption tax was not obviously regressive [7]. Qiong-Zhi Liu argue that Chinese personal income tax was regressive. It was not narrowing the income gap in redistribution phase [8].

Domestic scholars began late to research the relationship of tax and income distribution. Generally speaking, domestic research literature based on the existing tax theory abroad. They studied preference in the principle, system design and policy orientation. They used more qualitative analysis but less quantitative analysis. This was due to personal data was difficult to obtain. This paper, through the measurement model, analyzes the relationship between tax contribution and the income gap by using qualitative analysis and quantitative analysis.

\section{Definition of Tax Contribution}

Contribution rate refers to the ratio of effective or useful results to the number of occupied (consumed). It is an index that can be used to analyze the economic benefit. Specifically, contribution rate is the ratio of the amount of contribution to the amount of input, the ratio of the amount of output to the amount of consumption, the ratio of the amount of income to the amount of occupancy. According to definition of the contribution rate, we can define tax contribution as taxpayers have the ability to create tax in the case of occupying social resources. Tax contribution is a relative index. We need to consider the amount of social resources that are occupied by the taxpayer when we calculate tax contribution. The amount of social resources occupied by the taxpayer can be expressed by the gross domestic product. Tax contribution is the ratio of the total tax revenue to GDP. That is, tax contribution = the tax revenue/GDP. Tax contribution can be divided into turnover tax contribution, income tax contribution and property tax contribution. Turnover tax contribution is the ratio of turnover tax revenue to gross domestic product. Turnover tax include value added tax, business tax, consumption tax and tariffs; Income tax contribution is the ratio of income tax revenue to gross domestic product. Income tax includes enterprise in- 
come tax and personal income tax; property tax contribution is the ratio of property tax revenue to gross domestic product. Property tax includes real estate tax, land value-added tax, urban land use tax, travel tax and deed tax. Turnover tax contribution (income tax contribution or property tax contribution) $=$ turnover tax revenue (income tax revenue or property tax revenue)/GDP.

\section{Theoretical Analysis}

Analysis from the perspective of tax structure, turnover tax is easy to tax shifting. The added-value tax and business tax covers most of goods, including daily necessities. The elasticity of demand of daily necessities is small. The enterprises are easy to shift the tax burden on consumers by improving the commodity prices. Lowincome residents who bear the tax burden is higher than the high-income residents. It is not conducive to narrow the income gap. Consumption tax is mainly aimed at high income residents. It helps to adjust the income gap between residents. High income residents are mainly concentrated in cities. So it can adjust income gap between urban and rural areas. Therefore, it is not conducive to narrow the income gap between urban and rural areas by improving value added tax contribution and business tax contribution. It is conducive to narrow the income gap between urban and rural areas by improving consumption tax contribution. Value added tax and business tax are the most important part in the turnover tax. So it is not conducive to narrow the income gap between urban and rural areas by improving turnover tax contribution. Yi Liu analyzes turnover tax burden in different income groups. The empirical results show that turnover tax burden in different income groups is close to the proportion. So increase of turnover tax contribution is not conducive to narrowing the income gap [9]. Shaorong Li et al. (2006) according to the empirical test, it is concluded that increase of turnover tax contribution will expand the income gap between capital owners and labor owners. High income groups have more capital income, low income groups have less capital income. Labor income is the most important part of low income groups [10]. So turnover tax contribution will improve the income gap of residents. Ying Wan has the same point [7].

Whether the enterprise income tax can be used to adjust the income gap between urban and rural areas is controversial for the moment. Enterprise income tax affects the net profit of enterprises, reduces profit rate of capital. The capital of urban residents is high, the capital of rural residents is low. So it is beneficial to narrow the gap between urban and rural areas by improving enterprise income tax contribution. However, the private enterprise's life consumption is also easy to be included in the cost, resulting in the reduction of taxable income, which is not conducive to make income distribution fair. Personal income tax is recognized as one of the most effective means of adjusting income gap. Income distribution effects of personal income tax mainly come from progressive. Tax rate increases with increase of income level. It will help to reduce the income gap between high income and low income groups and achieve the purpose of adjusting income gap between urban and rural areas. But there are many defects in the current personal income tax system in China. For example, the tax rate of urban residents is far lower than the nominal tax, personal income tax of rural residents is lack, the structure of the individual income tax is unreasonable, the private economy of tax contribution is too low, tax evasion is serious, etc. It is not conducive to narrow the income gap between urban and rural areas by improving personal income tax contribution. Qiongzhi Liu found that the individual income tax has a certain degree of retirement. At the redistribution stage, improvement of tax contribution has not narrowed the degree of income gap [10]. Hua-Sheng Ouyang use micro data to study the personal income tax. The results show that individual income tax exists the phenomenon of "reverse adjustment". The growth of individual income tax contribution is not conducive to narrow residents' income gap [11].

Object of property tax is the taxpayer's owned or controlled property. Generally speaking, the higher income of residents is, the more the property is. Property income of urban residents is much higher than that of rural residents in China. Property tax can effectively adjust the income gap between urban and rural areas. Increase of property tax contribution will also help to narrow the income gap between urban and rural areas.

Structure of tax system is overall arrangement of various taxes of a country. And the main tax category plays a decisive role in overall function of tax system. Turnover tax is the main tax category of our country. Therefore, improvement of tax contribution will expand the income gap between urban and rural areas. Property tax, consumption tax and personal income tax play an important role for adjusting the residents income gap, but there are some important tax that are missing in the property tax. Consumption tax and personal income tax system is not reasonable. That is the reason why tax contribution cannot adjust income gap (Jian-Dong Chen, Xia Zhu soldier, 2011) [12].

Analysis from the perspective of the resident department revenue, the national income is allocated among the 
government, enterprises and residents. In primary distribution stage, the added value of residents in departments as a starting point, residents get labor remuneration by providing labor and get interest, rent, bonuses and other income by providing property. Labor remuneration is a major source of income of residents department, proportion of property income is very small. In the process of getting income, the residents department need pay production tax to government departments. Production tax is given priority to with turnover tax. Primary distribution stage emphasizes on efficiency principle. Production tax is not conducive to narrow income gap between residents. So the higher tax contribution, the greater income gap between residents. In the stage of redistribution, government departments use the income tax, transfer payments and other ways to adjust income share of the residents and the business sector. It could optimize the distribution pattern of national income. In terms of department of residents, government departments will receive income tax and social insurance from residents. At the same time, the government departments will transfer a portion of income to residents. Then it becomes the final formation of disposable income of residents. Redistribution pays great attention to fairness principle. Government department tax is conducive to narrow income gap between residents. So improvement of tax contribution is beneficial to narrow income gap between the residents.

\section{Empirical Analysis}

On the basis of above theoretical analysis, we use 2000-2014 panel data of 29 provinces and cities in our country (except Tibet) to establish econometric model for analysis.

\subsection{Setting of Econometric Model and Variable Selection}

In general, panel data has the characteristics of the time series, and it also can reflect the characteristics of the cross-section data. Panel data is widely used in econometric research. On the basis of our research, we set the following model:

Model 1: $I N E_{i t}=\alpha_{0}+\gamma_{1} P G D P_{i t}+\gamma_{2} P G D P_{i t}^{2}+\varepsilon_{i t}$.

Model 2: $\quad I N E_{i t}=\alpha_{0}+\gamma_{1} P G D P_{i t}+\gamma_{2} P G D P_{i t}^{2}+\beta_{1} C_{i t}+\beta_{2} S T R_{i t}+\gamma_{3} U R_{i t}+\gamma_{4} F I_{i t}+\gamma_{5} O P E N_{i t}+\varepsilon_{i t}$.

Model 3: $I N E_{i t}=\alpha_{0}+\gamma_{1} P G D P_{i t}+\gamma_{2} P G D P_{i t}^{2}+\beta_{1} L C_{i t}+\beta_{2} S C_{i t}+\beta_{3} C C_{i t}+\gamma_{4} F I_{i t}+\gamma_{5} O P E N_{i t}+\varepsilon_{i t}$.

Among them, $\alpha$ said intercept, $\beta$ and $\gamma$ said explanation variable coefficient, esaid random perturbation terms; the subscript $i$ said 29 provinces and cities $(i=1,2, \cdots, 29)$. The subscript t said time $(t=2000,2001, \cdots, 2014)$; INE said income gap between urban and rural areas, it is explained variable. Explanatory variable can be divided into core explanatory variable and other control variables, the specific variable settings and interpretation in Table 1.

Explained variable is income gap between urban and rural areas (INE). We use the ratio of urban to rural income to reflect. Core explanatory variable is tax contribution that is divided into two categories: one is reflecting

Table 1. Explained variable, core explanatory variable and other control variables.

\begin{tabular}{clrl}
\hline \multicolumn{1}{c}{ The variable name } & Symbol & \multicolumn{1}{c}{ Interpretation } \\
\hline Explained variable & $\begin{array}{l}\text { The income gap between urban } \\
\text { and rural areas }\end{array}$ & INE & $\begin{array}{l}\text { Urban per capita disposable income divided } \\
\text { by the per capita net income of rural residents }\end{array}$ \\
Core explanatory variable & $\begin{array}{l}\text { Tax contribution } \\
\text { Turnover tax contribution }\end{array}$ & LC & Tax revenue divided by GDP \\
& Income tax contribution & SC & Income tax revenue divided by GDP \\
& Property tax contribution & CC & Property tax revenue divided by GDP \\
Other control variables & economic development & PGDP & GDP per capita \\
& urbanization & UR & Urban population divided by the total population \\
& Fiscal spending & FI & The government fiscal spending divided by GDP \\
& Economic openness & OPEN & Import and export amount divided by GDP
\end{tabular}

The data source: China statistical yearbook, The Chinese tax yearbook. 
the tax revenue scale (C). The other one is reflecting structure of tax revenue, including turnover tax contribution (LC), income tax contribution (SC) and property tax contribution (CC). Turnover tax contribution is equal to the ratio of turnover tax revenue to GDP. Income tax contribution is equal to the ratio of income tax revenue to GDP. Property tax contribution is equal to the ratio of income tax revenue to GDP. In order to make econometric model results more convincing, we introduce other control variables to the model. Other control variables include economic development (PGDP), urbanization (UR), fiscal expenditure (FI) and economic openness (OPEN). Economic development is equal to GDP per capita. Urbanization is equal to urban population divided by the total population. Fiscal expenditure is equal to government fiscal spending divided by GDP. Economic openness is equal to import and export amount divided by GDP.

\subsection{Data Source}

The data of urban per capita disposable income, rural per capita net income, GDP, proportion of urban population, total population, total import and export amount, and government fiscal spending are from 2000-2014 China statistical yearbook [13], part of data are from the statistical yearbook of provinces and cities. The data of total tax revenues, turnover tax, income tax and property tax are from 2000-2014 Chinese tax yearbook [14]. Tables 2-10 show the value of each variable.

Table 2. The value of the income gap between urban and rural areas. Unit: yuan.

\begin{tabular}{|c|c|c|c|c|c|c|c|c|}
\hline & 2000 & 2001 & 2002 & 2003 & 2004 & 2005 & 2006 & 2007 \\
\hline Beijing & 2.25 & 2.30 & 2.31 & 2.48 & 2.53 & 2.40 & 2.41 & 2.33 \\
\hline Tianjin & 2.25 & 2.27 & 2.18 & 2.26 & 2.28 & 2.27 & 2.29 & 2.33 \\
\hline Hebei & 2.28 & 2.30 & 2.49 & 2.54 & 2.51 & 2.62 & 2.71 & 2.72 \\
\hline Shanxi & 2.48 & 2.76 & 2.90 & 3.05 & 3.05 & 3.08 & 3.15 & 3.15 \\
\hline Inner Mongolia & 2.52 & 2.81 & 2.90 & 3.09 & 3.12 & 3.06 & 3.10 & 3.13 \\
\hline Liaoning & 2.27 & 2.27 & 2.37 & 2.47 & 2.42 & 2.47 & 2.54 & 2.58 \\
\hline Jilin & 2.38 & 2.45 & 2.72 & 2.77 & 2.61 & 2.66 & 2.68 & 2.69 \\
\hline Heilongjiang & 2.29 & 2.38 & 2.54 & 2.66 & 2.49 & 2.57 & 2.58 & 2.48 \\
\hline Shanghai & 2.09 & 2.19 & 2.13 & 2.23 & 2.36 & 2.26 & 2.26 & 2.33 \\
\hline Jiangsu & 1.89 & 1.95 & 2.05 & 2.18 & 2.20 & 2.33 & 2.42 & 2.50 \\
\hline Zhejiang & 2.18 & 2.28 & 2.37 & 2.45 & 2.45 & 2.45 & 2.49 & 2.49 \\
\hline Anhui & 2.74 & 2.81 & 2.85 & 3.19 & 3.01 & 3.21 & 3.29 & 3.23 \\
\hline Fujian & 2.30 & 2.46 & 2.60 & 2.68 & 2.73 & 2.77 & 2.84 & 2.84 \\
\hline Jiangxi & 2.39 & 2.47 & 2.75 & 2.81 & 2.71 & 2.75 & 2.76 & 2.83 \\
\hline Shandong & 2.44 & 2.53 & 2.58 & 2.67 & 2.69 & 2.73 & 2.79 & 2.86 \\
\hline Henan & 2.40 & 2.51 & 2.82 & 3.10 & 3.02 & 3.02 & 3.01 & 2.98 \\
\hline Hubei & 2.44 & 2.49 & 2.78 & 2.85 & 2.78 & 2.83 & 2.87 & 2.87 \\
\hline Hunan & 2.83 & 2.95 & 2.90 & 3.03 & 3.04 & 3.05 & 3.10 & 3.15 \\
\hline Guangdong & 2.67 & 2.76 & 2.85 & 3.05 & 3.12 & 3.15 & 3.15 & 3.15 \\
\hline Guangxi & 3.13 & 3.43 & 3.63 & 3.72 & 3.77 & 3.72 & 3.57 & 3.78 \\
\hline Hainan & 2.46 & 2.62 & 2.82 & 2.80 & 2.75 & 2.70 & 2.89 & 2.90 \\
\hline Sichuan & 3.10 & 3.20 & 3.14 & 3.16 & 3.06 & 2.99 & 3.11 & 3.13 \\
\hline Guizhou & 3.73 & 3.86 & 3.99 & 4.20 & 4.25 & 4.34 & 4.59 & 4.50 \\
\hline Yunnan & 4.28 & 4.43 & 4.50 & 4.50 & 4.76 & 4.54 & 4.47 & 4.36 \\
\hline Shaanxi & 3.55 & 3.68 & 3.97 & 4.06 & 4.01 & 4.03 & 4.10 & 4.07 \\
\hline Gansu & 3.44 & 3.57 & 3.87 & 3.98 & 3.98 & 4.08 & 4.18 & 4.30 \\
\hline Qinghai & 3.47 & 3.76 & 3.70 & 3.76 & 3.74 & 3.75 & 3.82 & 3.83 \\
\hline Ningxia & 2.85 & 3.04 & 3.16 & 3.20 & 3.11 & 3.23 & 3.32 & 3.41 \\
\hline Xinjiang & 3.49 & 3.74 & 3.70 & 3.41 & 3.34 & 3.22 & 3.24 & 3.24 \\
\hline
\end{tabular}




\section{Continued}

\begin{tabular}{|c|c|c|c|c|c|c|c|}
\hline & 2008 & 2009 & 2010 & 2011 & 2012 & 2013 & 2014 \\
\hline Beijing & 2.32 & 2.29 & 2.19 & 2.23 & 2.21 & 2.20 & 2.18 \\
\hline Tianjin & 2.46 & 2.46 & 2.41 & 2.18 & 2.11 & 2.04 & 2.06 \\
\hline Hebei & 2.80 & 2.86 & 2.73 & 2.57 & 2.54 & 2.48 & 2.44 \\
\hline Shanxi & 3.20 & 3.30 & 3.30 & 3.24 & 3.21 & 3.14 & 3.04 \\
\hline Inner Mongolia & 3.10 & 3.21 & 3.20 & 3.07 & 3.04 & 2.97 & 2.98 \\
\hline Liaoning & 2.58 & 2.65 & 2.56 & 2.47 & 2.47 & 2.43 & 2.51 \\
\hline Jilin & 2.60 & 2.66 & 2.47 & 2.37 & 2.35 & 2.32 & 2.22 \\
\hline Heilongjiang & 2.39 & 2.41 & 2.23 & 2.07 & 2.06 & 2.03 & 2.02 \\
\hline Shanghai & 2.33 & 2.31 & 2.28 & 2.26 & 2.26 & 2.24 & 2.22 \\
\hline Jiangsu & 2.54 & 2.57 & 2.52 & 2.44 & 2.43 & 2.39 & 2.33 \\
\hline Zhejiang & 2.45 & 2.46 & 2.42 & 2.37 & 2.37 & 2.35 & 2.31 \\
\hline Anhui & 3.09 & 3.13 & 2.99 & 2.99 & 2.94 & 2.85 & 2.81 \\
\hline Fujian & 2.90 & 2.93 & 2.93 & 2.84 & 2.81 & 2.76 & 2.75 \\
\hline Jiangxi & 2.74 & 2.76 & 2.67 & 2.54 & 2.54 & 2.49 & 2.43 \\
\hline Shandong & 2.89 & 2.91 & 2.85 & 2.73 & 2.73 & 2.66 & 2.64 \\
\hline Henan & 2.97 & 2.99 & 2.88 & 2.76 & 2.72 & 2.64 & 2.61 \\
\hline Hubei & 2.82 & 2.85 & 2.75 & 2.66 & 2.65 & 2.58 & 2.55 \\
\hline Hunan & 3.06 & 3.07 & 2.95 & 2.87 & 2.87 & 2.80 & 2.77 \\
\hline Guangdong & 3.08 & 3.12 & 3.03 & 2.87 & 2.87 & 2.84 & 2.83 \\
\hline Guangxi & 3.83 & 3.88 & 3.76 & 3.60 & 3.54 & 3.43 & 3.41 \\
\hline Hainan & 2.87 & 2.90 & 2.95 & 2.85 & 2.82 & 2.75 & 2.73 \\
\hline Sichuan & 3.07 & 3.10 & 3.04 & 2.92 & 2.90 & 2.83 & 2.83 \\
\hline Guizhou & 4.20 & 4.28 & 4.07 & 3.98 & 3.93 & 3.80 & 3.73 \\
\hline Yunnan & 4.27 & 4.28 & 4.06 & 3.93 & 3.89 & 3.78 & 3.75 \\
\hline Shaanxi & 4.10 & 4.11 & 3.82 & 3.63 & 3.60 & 3.52 & 3.50 \\
\hline Gansu & 4.03 & 4.00 & 3.85 & 3.83 & 3.81 & 3.71 & 3.70 \\
\hline Qinghai & 3.80 & 3.79 & 3.59 & 3.39 & 3.27 & 3.15 & 3.13 \\
\hline Ningxia & 3.51 & 3.46 & 3.28 & 3.25 & 3.21 & 3.15 & 3.12 \\
\hline Xinjiang & 3.26 & 3.16 & 2.94 & 2.85 & 2.80 & 2.72 & 2.71 \\
\hline
\end{tabular}

Table 11 is descriptive statistics of each variable.

\subsection{Estimation Method and Measurement Test Results}

We use the reviews 6.0 software and choose LLC test, ADF-F test and PP-F test for unit root test of the variables. Due to space limitations, we do not list each variable inspection process. Results of unit root test show that all variables are significant. We could suggest that each variable is zero order single whole and don't need to do cointegration test.

Panel data model can generally be divided into three categories: mixed regression model, fixed effects regression model and random effects regression model. In order to determine what kind of panel data model to use. We use the likelihood ratio test and Hausman test to determine model type. Likelihood ratio test is used to select the fixed effects regression model or mixed regression model. The null hypothesis choose mixed regression 
Table 3. The value of tax contribution.

\begin{tabular}{|c|c|c|c|c|c|c|c|c|}
\hline & 2000 & 2001 & 2002 & 2003 & 2004 & 2005 & 2006 & 2007 \\
\hline Beijing & 0.36 & 0.43 & 0.42 & 0.42 & 0.44 & 0.37 & 0.42 & 0.46 \\
\hline Tianjin & 0.20 & 0.23 & 0.24 & 0.25 & 0.27 & 0.28 & 0.30 & 0.32 \\
\hline Hebei & 0.07 & 0.08 & 0.08 & 0.08 & 0.08 & 0.10 & 0.10 & 0.11 \\
\hline Shanxi & 0.11 & 0.13 & 0.13 & 0.14 & 0.16 & 0.17 & 0.18 & 0.19 \\
\hline Inner Mongolia & 0.09 & 0.09 & 0.10 & 0.10 & 0.11 & 0.12 & 0.12 & 0.13 \\
\hline Liaoning & 0.11 & 0.14 & 0.14 & 0.14 & 0.15 & 0.16 & 0.16 & 0.16 \\
\hline Jilin & 0.10 & 0.11 & 0.12 & 0.12 & 0.12 & 0.11 & 0.11 & 0.11 \\
\hline Heilongjiang & 0.11 & 0.12 & 0.12 & 0.11 & 0.12 & 0.13 & 0.14 & 0.13 \\
\hline Shanghai & 0.31 & 0.33 & 0.34 & 0.39 & 0.42 & 0.38 & 0.40 & 0.53 \\
\hline Jiangsu & 0.09 & 0.12 & 0.13 & 0.14 & 0.15 & 0.15 & 0.15 & 0.17 \\
\hline Zhejiang & 0.12 & 0.16 & 0.16 & 0.17 & 0.19 & 0.19 & 0.19 & 0.20 \\
\hline Anhui & 0.07 & 0.08 & 0.08 & 0.09 & 0.09 & 0.10 & 0.11 & 0.11 \\
\hline Fujian & 0.09 & 0.11 & 0.11 & 0.08 & 0.12 & 0.13 & 0.14 & 0.14 \\
\hline Jiangxi & 0.07 & 0.08 & 0.07 & 0.08 & 0.08 & 0.08 & 0.09 & 0.10 \\
\hline Shandong & 0.09 & 0.11 & 0.10 & 0.10 & 0.10 & 0.11 & 0.12 & 0.12 \\
\hline Henan & 0.07 & 0.07 & 0.07 & 0.07 & 0.07 & 0.08 & 0.08 & 0.09 \\
\hline Hubei & 0.07 & 0.07 & 0.08 & 0.08 & 0.08 & 0.11 & 0.11 & 0.11 \\
\hline Hunan & 0.07 & 0.08 & 0.08 & 0.08 & 0.09 & 0.09 & 0.10 & 0.10 \\
\hline Guangdong & 0.19 & 0.21 & 0.22 & 0.22 & 0.22 & 0.19 & 0.19 & 0.22 \\
\hline Guangxi & 0.09 & 0.10 & 0.10 & 0.10 & 0.10 & 0.10 & 0.10 & 0.10 \\
\hline Hainan & 0.08 & 0.10 & 0.10 & 0.11 & 0.12 & 0.13 & 0.14 & 0.17 \\
\hline Sichuan & 0.08 & 0.09 & 0.09 & 0.09 & 0.09 & 0.10 & 0.11 & 0.12 \\
\hline Guizhou & 0.13 & 0.13 & 0.14 & 0.14 & 0.16 & 0.16 & 0.17 & 0.17 \\
\hline Yunnan & 0.21 & 0.20 & 0.20 & 0.20 & 0.20 & 0.20 & 0.21 & 0.22 \\
\hline Shaanxi & 0.11 & 0.12 & 0.12 & 0.12 & 0.13 & 0.13 & 0.14 & 0.15 \\
\hline Gansu & 0.10 & 0.11 & 0.12 & 0.12 & 0.12 & 0.12 & 0.12 & 0.13 \\
\hline Qinghai & 0.09 & 0.10 & 0.10 & 0.10 & 0.10 & 0.11 & 0.12 & 0.13 \\
\hline Ningxia & 0.11 & 0.13 & 0.12 & 0.12 & 0.14 & 0.13 & 0.13 & 0.14 \\
\hline Xinjiang & 0.11 & 0.12 & 0.12 & 0.12 & 0.14 & 0.15 & 0.16 & 0.17 \\
\hline
\end{tabular}




\section{Continued}

\begin{tabular}{|c|c|c|c|c|c|c|c|}
\hline & 2008 & 2009 & 2010 & 2011 & 2012 & 2013 & 2014 \\
\hline Beijing & 0.50 & 0.51 & 0.44 & 0.48 & 0.51 & 0.53 & 0.58 \\
\hline Tianjin & 0.32 & 0.27 & 0.30 & 0.30 & 0.29 & 0.28 & 0.33 \\
\hline Hebei & 0.11 & 0.11 & 0.12 & 0.12 & 0.13 & 0.13 & 0.18 \\
\hline Shanxi & 0.21 & 0.19 & 0.18 & 0.18 & 0.19 & 0.18 & 0.23 \\
\hline Inner Mongolia & 0.13 & 0.12 & 0.13 & 0.14 & 0.14 & 0.14 & 0.19 \\
\hline Liaoning & 0.17 & 0.17 & 0.18 & 0.18 & 0.19 & 0.18 & 0.23 \\
\hline Jilin & 0.12 & 0.12 & 0.12 & 0.13 & 0.14 & 0.15 & 0.20 \\
\hline Heilongjiang & 0.13 & 0.13 & 0.13 & 0.14 & 0.15 & 0.15 & 0.20 \\
\hline Shanghai & 0.48 & 0.44 & 0.47 & 0.50 & 0.52 & 0.51 & 0.56 \\
\hline Jiangsu & 0.17 & 0.17 & 0.17 & 0.18 & 0.19 & 0.19 & 0.24 \\
\hline Zhejiang & 0.21 & 0.20 & 0.06 & 0.21 & 0.22 & 0.22 & 0.27 \\
\hline Anhui & 0.12 & 0.12 & 0.13 & 0.14 & 0.15 & 0.15 & 0.20 \\
\hline Fujian & 0.15 & 0.14 & 0.15 & 0.15 & 0.16 & 0.17 & 0.22 \\
\hline Jiangxi & 0.10 & 0.11 & 0.12 & 0.12 & 0.14 & 0.14 & 0.19 \\
\hline Shandong & 0.12 & 0.12 & 0.10 & 0.14 & 0.15 & 0.14 & 0.19 \\
\hline Henan & 0.08 & 0.08 & 0.08 & 0.09 & 0.10 & 0.10 & 0.15 \\
\hline Hubei & 0.11 & 0.11 & 0.11 & 0.11 & 0.13 & 0.13 & 0.18 \\
\hline Hunan & 0.10 & 0.09 & 0.09 & 0.10 & 0.11 & 0.11 & 0.16 \\
\hline Guangdong & 0.22 & 0.21 & 0.22 & 0.22 & 0.23 & 0.23 & 0.28 \\
\hline Guangxi & 0.10 & 0.10 & 0.11 & 0.12 & 0.13 & 0.13 & 0.18 \\
\hline Hainan & 0.19 & 0.21 & 0.23 & 0.25 & 0.25 & 0.24 & 0.29 \\
\hline Sichuan & 0.12 & 0.12 & 0.13 & 0.13 & 0.14 & 0.15 & 0.20 \\
\hline Guizhou & 0.17 & 0.17 & 0.18 & 0.18 & 0.19 & 0.19 & 0.24 \\
\hline Yunnan & 0.22 & 0.22 & 0.23 & 0.22 & 0.23 & 0.23 & 0.28 \\
\hline Shaanxi & 0.15 & 0.15 & 0.16 & 0.17 & 0.17 & 0.16 & 0.21 \\
\hline Gansu & 0.12 & 0.15 & 0.15 & 0.15 & 0.16 & 0.15 & 0.20 \\
\hline Qinghai & 0.14 & 0.15 & 0.15 & 0.15 & 0.16 & 0.16 & 0.21 \\
\hline Ningxia & 0.15 & 0.14 & 0.15 & 0.15 & 0.17 & 0.18 & 0.23 \\
\hline Xinjiang & 0.18 & 0.19 & 0.20 & 0.22 & 0.22 & 0.21 & 0.26 \\
\hline
\end{tabular}


Table 4. The value of turnover tax contribution.

\begin{tabular}{|c|c|c|c|c|c|c|c|c|}
\hline & 2000 & 2001 & 2002 & 2003 & 2004 & 2005 & 2006 & 2007 \\
\hline Beijing & 0.19 & 0.22 & 0.22 & 0.22 & 0.23 & 0.16 & 0.18 & 0.18 \\
\hline Tianjin & 0.16 & 0.17 & 0.18 & 0.19 & 0.20 & 0.20 & 0.21 & 0.22 \\
\hline Hebei & 0.05 & 0.05 & 0.05 & 0.05 & 0.06 & 0.07 & 0.07 & 0.07 \\
\hline Shanxi & 0.08 & 0.09 & 0.10 & 0.10 & 0.12 & 0.12 & 0.12 & 0.12 \\
\hline Inner Mongolia & 0.07 & 0.07 & 0.07 & 0.08 & 0.09 & 0.09 & 0.09 & 0.09 \\
\hline Liaoning & 0.06 & 0.06 & 0.07 & 0.07 & 0.07 & 0.07 & 0.07 & 0.07 \\
\hline Jilin & 0.07 & 0.08 & 0.09 & 0.09 & 0.09 & 0.08 & 0.08 & 0.08 \\
\hline Heilongjiang & 0.08 & 0.08 & 0.08 & 0.07 & 0.08 & 0.08 & 0.09 & 0.08 \\
\hline Shanghai & 0.19 & 0.22 & 0.24 & 0.28 & 0.29 & 0.26 & 0.27 & 0.28 \\
\hline Jiangsu & 0.07 & 0.09 & 0.09 & 0.10 & 0.11 & 0.11 & 0.11 & 0.11 \\
\hline Zhejiang & 0.06 & 0.07 & 0.08 & 0.08 & 0.08 & 0.08 & 0.08 & 0.09 \\
\hline Anhui & 0.05 & 0.06 & 0.06 & 0.06 & 0.06 & 0.07 & 0.08 & 0.08 \\
\hline Fujian & 0.04 & 0.05 & 0.05 & 0.06 & 0.06 & 0.06 & 0.06 & 0.06 \\
\hline Jiangxi & 0.05 & 0.05 & 0.05 & 0.06 & 0.06 & 0.06 & 0.06 & 0.07 \\
\hline Shandong & 0.05 & 0.05 & 0.05 & 0.05 & 0.05 & 0.05 & 0.06 & 0.06 \\
\hline Henan & 0.05 & 0.05 & 0.05 & 0.05 & 0.05 & 0.05 & 0.05 & 0.05 \\
\hline Hubei & 0.05 & 0.05 & 0.05 & 0.06 & 0.06 & 0.07 & 0.07 & 0.07 \\
\hline Hunan & 0.05 & 0.06 & 0.06 & 0.06 & 0.07 & 0.07 & 0.07 & 0.07 \\
\hline Guangdong & 0.09 & 0.11 & 0.11 & 0.11 & 0.12 & 0.10 & 0.10 & 0.10 \\
\hline Guangxi & 0.06 & 0.07 & 0.07 & 0.07 & 0.07 & 0.07 & 0.07 & 0.07 \\
\hline Hainan & 0.05 & 0.07 & 0.07 & 0.08 & 0.09 & 0.09 & 0.10 & 0.13 \\
\hline Sichuan & 0.06 & 0.06 & 0.07 & 0.07 & 0.07 & 0.07 & 0.07 & 0.08 \\
\hline Guizhou & 0.10 & 0.10 & 0.11 & 0.11 & 0.11 & 0.11 & 0.12 & 0.11 \\
\hline Yunnan & 0.16 & 0.15 & 0.15 & 0.15 & 0.15 & 0.15 & 0.15 & 0.15 \\
\hline Shaanxi & 0.08 & 0.09 & 0.09 & 0.09 & 0.10 & 0.09 & 0.10 & 0.10 \\
\hline Gansu & 0.08 & 0.08 & 0.09 & 0.09 & 0.10 & 0.09 & 0.09 & 0.10 \\
\hline Qinghai & 0.07 & 0.07 & 0.08 & 0.08 & 0.08 & 0.08 & 0.09 & 0.10 \\
\hline Ningxia & 0.08 & 0.09 & 0.09 & 0.09 & 0.10 & 0.10 & 0.10 & 0.10 \\
\hline Xinjiang & 0.07 & 0.08 & 0.09 & 0.09 & 0.10 & 0.11 & 0.11 & 0.12 \\
\hline
\end{tabular}




\section{Continued}

\begin{tabular}{|c|c|c|c|c|c|c|c|}
\hline & 2008 & 2009 & 2010 & 2011 & 2012 & 2013 & 2014 \\
\hline Beijing & 0.18 & 0.17 & 0.17 & 0.17 & 0.17 & 0.17 & 0.19 \\
\hline Tianjin & 0.22 & 0.20 & 0.22 & 0.21 & 0.20 & 0.19 & 0.21 \\
\hline Hebei & 0.07 & 0.08 & 0.08 & 0.08 & 0.08 & 0.08 & 0.10 \\
\hline Shanxi & 0.14 & 0.12 & 0.12 & 0.12 & 0.11 & 0.10 & 0.12 \\
\hline Inner Mongolia & 0.09 & 0.08 & 0.08 & 0.08 & 0.08 & 0.07 & 0.09 \\
\hline Liaoning & 0.07 & 0.07 & 0.07 & 0.07 & 0.07 & 0.06 & 0.08 \\
\hline Jilin & 0.08 & 0.08 & 0.08 & 0.09 & 0.08 & 0.08 & 0.10 \\
\hline Heilongjiang & 0.08 & 0.08 & 0.08 & 0.09 & 0.09 & 0.08 & 0.10 \\
\hline Shanghai & 0.27 & 0.28 & 0.30 & 0.32 & 0.32 & 0.31 & 0.33 \\
\hline Jiangsu & 0.11 & 0.11 & 0.11 & 0.11 & 0.11 & 0.11 & 0.13 \\
\hline Zhejiang & 0.09 & 0.09 & 0.09 & 0.09 & 0.09 & 0.09 & 0.11 \\
\hline Anhui & 0.08 & 0.08 & 0.09 & 0.09 & 0.08 & 0.08 & 0.10 \\
\hline Fujian & 0.06 & 0.06 & 0.07 & 0.07 & 0.07 & 0.07 & 0.09 \\
\hline Jiangxi & 0.07 & 0.07 & 0.08 & 0.08 & 0.08 & 0.08 & 0.10 \\
\hline Shandong & 0.06 & 0.06 & 0.07 & 0.07 & 0.07 & 0.07 & 0.09 \\
\hline Henan & 0.05 & 0.05 & 0.05 & 0.05 & 0.05 & 0.05 & 0.07 \\
\hline Hubei & 0.07 & 0.07 & 0.07 & 0.07 & 0.07 & 0.07 & 0.09 \\
\hline Hunan & 0.07 & 0.07 & 0.07 & 0.07 & 0.07 & 0.07 & 0.09 \\
\hline Guangdong & 0.10 & 0.10 & 0.10 & 0.10 & 0.11 & 0.10 & 0.12 \\
\hline Guangxi & 0.07 & 0.07 & 0.08 & 0.08 & 0.08 & 0.08 & 0.10 \\
\hline Hainan & 0.14 & 0.15 & 0.16 & 0.16 & 0.15 & 0.13 & 0.15 \\
\hline Sichuan & 0.07 & 0.07 & 0.08 & 0.08 & 0.08 & 0.08 & 0.10 \\
\hline Guizhou & 0.11 & 0.11 & 0.11 & 0.11 & 0.11 & 0.11 & 0.13 \\
\hline Yunnan & 0.15 & 0.15 & 0.16 & 0.15 & 0.15 & 0.14 & 0.16 \\
\hline Shaanxi & 0.10 & 0.10 & 0.11 & 0.11 & 0.10 & 0.10 & 0.12 \\
\hline Gansu & 0.09 & 0.11 & 0.11 & 0.11 & 0.11 & 0.10 & 0.12 \\
\hline Qinghai & 0.09 & 0.10 & 0.10 & 0.10 & 0.10 & 0.10 & 0.12 \\
\hline Ningxia & 0.10 & 0.10 & 0.10 & 0.09 & 0.11 & 0.11 & 0.13 \\
\hline Xinjiang & 0.12 & 0.13 & 0.14 & 0.14 & 0.14 & 0.13 & 0.15 \\
\hline
\end{tabular}


Table 5. The value of income tax contribution.

\begin{tabular}{|c|c|c|c|c|c|c|c|c|}
\hline & 2000 & 2001 & 2002 & 2003 & 2004 & 2005 & 2006 & 2007 \\
\hline Beijing & 0.14 & 0.18 & 0.18 & 0.18 & 0.19 & 0.19 & 0.23 & 0.25 \\
\hline Tianjin & 0.04 & 0.06 & 0.05 & 0.05 & 0.06 & 0.07 & 0.08 & 0.08 \\
\hline Hebei & 0.01 & 0.02 & 0.02 & 0.02 & 0.02 & 0.02 & 0.02 & 0.02 \\
\hline Shanxi & 0.02 & 0.02 & 0.02 & 0.02 & 0.03 & 0.03 & 0.04 & 0.05 \\
\hline Inner Mongolia & 0.01 & 0.02 & 0.02 & 0.01 & 0.02 & 0.02 & 0.02 & 0.02 \\
\hline Liaoning & 0.02 & 0.03 & 0.02 & 0.02 & 0.03 & 0.03 & 0.03 & 0.03 \\
\hline Jilin & 0.02 & 0.02 & 0.02 & 0.02 & 0.02 & 0.02 & 0.02 & 0.02 \\
\hline Heilongjiang & 0.03 & 0.03 & 0.03 & 0.03 & 0.03 & 0.04 & 0.04 & 0.04 \\
\hline Shanghai & 0.06 & 0.07 & 0.08 & 0.07 & 0.10 & 0.10 & 0.10 & 0.13 \\
\hline Jiangsu & 0.02 & 0.03 & 0.03 & 0.03 & 0.03 & 0.03 & 0.03 & 0.04 \\
\hline Zhejiang & 0.03 & 0.04 & 0.04 & 0.04 & 0.04 & 0.04 & 0.04 & 0.05 \\
\hline Anhui & 0.01 & 0.02 & 0.02 & 0.02 & 0.02 & 0.02 & 0.02 & 0.02 \\
\hline Fujian & 0.02 & 0.03 & 0.02 & 0.03 & 0.03 & 0.03 & 0.03 & 0.03 \\
\hline Jiangxi & 0.01 & 0.02 & 0.01 & 0.01 & 0.02 & 0.02 & 0.02 & 0.02 \\
\hline Shandong & 0.02 & 0.03 & 0.02 & 0.02 & 0.02 & 0.02 & 0.02 & 0.02 \\
\hline Henan & 0.01 & 0.02 & 0.02 & 0.02 & 0.02 & 0.02 & 0.02 & 0.02 \\
\hline Hubei & 0.01 & 0.02 & 0.02 & 0.02 & 0.02 & 0.03 & 0.03 & 0.03 \\
\hline Hunan & 0.01 & 0.01 & 0.01 & 0.01 & 0.01 & 0.01 & 0.02 & 0.02 \\
\hline Guangdong & 0.03 & 0.04 & 0.04 & 0.04 & 0.04 & 0.04 & 0.04 & 0.05 \\
\hline Guangxi & 0.02 & 0.03 & 0.02 & 0.02 & 0.02 & 0.02 & 0.02 & 0.02 \\
\hline Hainan & 0.02 & 0.02 & 0.02 & 0.02 & 0.02 & 0.02 & 0.02 & 0.03 \\
\hline Sichuan & 0.01 & 0.02 & 0.02 & 0.01 & 0.02 & 0.02 & 0.02 & 0.03 \\
\hline Guizhou & 0.02 & 0.02 & 0.02 & 0.02 & 0.03 & 0.03 & 0.03 & 0.04 \\
\hline Yunnan & 0.03 & 0.03 & 0.03 & 0.03 & 0.03 & 0.03 & 0.03 & 0.04 \\
\hline Shaanxi & 0.02 & 0.03 & 0.02 & 0.02 & 0.02 & 0.02 & 0.03 & 0.03 \\
\hline Gansu & 0.01 & 0.02 & 0.02 & 0.02 & 0.02 & 0.02 & 0.02 & 0.02 \\
\hline Qinghai & 0.02 & 0.02 & 0.01 & 0.01 & 0.01 & 0.02 & 0.02 & 0.02 \\
\hline Ningxia & 0.02 & 0.03 & 0.02 & 0.02 & 0.02 & 0.02 & 0.02 & 0.02 \\
\hline Xinjiang & 0.02 & 0.03 & 0.02 & 0.02 & 0.02 & 0.03 & 0.03 & 0.03 \\
\hline
\end{tabular}




\section{Continued}

\begin{tabular}{|c|c|c|c|c|c|c|c|}
\hline & 2008 & 2009 & 2010 & 2011 & 2012 & 2013 & 2014 \\
\hline Beijing & 0.30 & 0.30 & 0.24 & 0.27 & 0.29 & 0.31 & 0.32 \\
\hline Tianjin & 0.08 & 0.06 & 0.06 & 0.07 & 0.06 & 0.06 & 0.07 \\
\hline Hebei & 0.03 & 0.02 & 0.02 & 0.03 & 0.03 & 0.02 & 0.03 \\
\hline Shanxi & 0.04 & 0.05 & 0.04 & 0.05 & 0.05 & 0.05 & 0.05 \\
\hline Inner Mongolia & 0.03 & 0.03 & 0.03 & 0.04 & 0.04 & 0.03 & 0.03 \\
\hline Liaoning & 0.03 & 0.02 & 0.02 & 0.02 & 0.02 & 0.02 & 0.02 \\
\hline Jilin & 0.03 & 0.03 & 0.03 & 0.03 & 0.03 & 0.03 & 0.04 \\
\hline Heilongjiang & 0.04 & 0.03 & 0.03 & 0.04 & 0.04 & 0.04 & 0.05 \\
\hline Shanghai & 0.14 & 0.12 & 0.13 & 0.14 & 0.14 & 0.14 & 0.15 \\
\hline Jiangsu & 0.04 & 0.04 & 0.04 & 0.05 & 0.04 & 0.04 & 0.05 \\
\hline Zhejiang & 0.04 & 0.03 & 0.04 & 0.04 & 0.04 & 0.04 & 0.04 \\
\hline Anhui & 0.03 & 0.02 & 0.03 & 0.03 & 0.03 & 0.03 & 0.04 \\
\hline Fujian & 0.03 & 0.03 & 0.03 & 0.03 & 0.03 & 0.03 & 0.04 \\
\hline Jiangxi & 0.02 & 0.02 & 0.02 & 0.03 & 0.03 & 0.03 & 0.03 \\
\hline Shandong & 0.02 & 0.02 & 0.02 & 0.02 & 0.02 & 0.02 & 0.02 \\
\hline Henan & 0.02 & 0.02 & 0.02 & 0.02 & 0.02 & 0.02 & 0.03 \\
\hline Hubei & 0.03 & 0.03 & 0.02 & 0.03 & 0.03 & 0.03 & 0.03 \\
\hline Hunan & 0.02 & 0.02 & 0.02 & 0.02 & 0.02 & 0.02 & 0.02 \\
\hline Guangdong & 0.04 & 0.03 & 0.04 & 0.04 & 0.03 & 0.04 & 0.04 \\
\hline Guangxi & 0.02 & 0.02 & 0.02 & 0.02 & 0.02 & 0.02 & 0.03 \\
\hline Hainan & 0.04 & 0.04 & 0.04 & 0.05 & 0.05 & 0.05 & 0.06 \\
\hline Sichuan & 0.03 & 0.03 & 0.03 & 0.03 & 0.03 & 0.03 & 0.04 \\
\hline Guizhou & 0.04 & 0.04 & 0.04 & 0.04 & 0.04 & 0.04 & 0.05 \\
\hline Yunnan & 0.04 & 0.04 & 0.04 & 0.04 & 0.04 & 0.04 & 0.04 \\
\hline Shaanxi & 0.04 & 0.03 & 0.03 & 0.04 & 0.04 & 0.04 & 0.04 \\
\hline Gansu & 0.02 & 0.02 & 0.02 & 0.02 & 0.02 & 0.02 & 0.03 \\
\hline Qinghai & 0.03 & 0.03 & 0.03 & 0.03 & 0.03 & 0.03 & 0.04 \\
\hline Ningxia & 0.02 & 0.03 & 0.03 & 0.04 & 0.03 & 0.03 & 0.04 \\
\hline Xinjiang & 0.04 & 0.03 & 0.04 & 0.05 & 0.05 & 0.05 & 0.05 \\
\hline
\end{tabular}


Table 6. The value of property tax contribution.

\begin{tabular}{|c|c|c|c|c|c|c|c|c|}
\hline & 2000 & 2001 & 2002 & 2003 & 2004 & 2005 & 2006 & 2007 \\
\hline Beijing & 0.010 & 0.009 & 0.010 & 0.011 & 0.009 & 0.006 & 0.007 & 0.011 \\
\hline Tianjin & 0.004 & 0.003 & 0.004 & 0.004 & 0.003 & 0.003 & 0.004 & 0.005 \\
\hline Hebei & 0.002 & 0.002 & 0.002 & 0.002 & 0.002 & 0.002 & 0.002 & 0.003 \\
\hline Shanxi & 0.004 & 0.004 & 0.004 & 0.003 & 0.003 & 0.003 & 0.003 & 0.003 \\
\hline Inner Mongolia & 0.006 & 0.005 & 0.005 & 0.005 & 0.005 & 0.004 & 0.005 & 0.006 \\
\hline Liaoning & 0.004 & 0.004 & 0.004 & 0.004 & 0.004 & 0.004 & 0.005 & 0.008 \\
\hline Jilin & 0.003 & 0.003 & 0.003 & 0.004 & 0.004 & 0.003 & 0.003 & 0.004 \\
\hline Heilongjiang & 0.003 & 0.003 & 0.003 & 0.003 & 0.003 & 0.003 & 0.003 & 0.003 \\
\hline Shanghai & 0.004 & 0.004 & 0.004 & 0.004 & 0.005 & 0.007 & 0.008 & 0.008 \\
\hline Jiangsu & 0.002 & 0.002 & 0.002 & 0.003 & 0.003 & 0.004 & 0.004 & 0.006 \\
\hline Zhejiang & 0.002 & 0.002 & 0.002 & 0.002 & 0.003 & 0.004 & 0.004 & 0.005 \\
\hline Anhui & 0.003 & 0.003 & 0.003 & 0.003 & 0.003 & 0.003 & 0.003 & 0.004 \\
\hline Fujian & 0.002 & 0.002 & 0.002 & 0.003 & 0.003 & 0.003 & 0.004 & 0.004 \\
\hline Jiangxi & 0.002 & 0.002 & 0.002 & 0.002 & 0.002 & 0.002 & 0.003 & 0.004 \\
\hline Shandong & 0.003 & 0.003 & 0.003 & 0.004 & 0.003 & 0.004 & 0.004 & 0.005 \\
\hline Henan & 0.002 & 0.002 & 0.002 & 0.002 & 0.002 & 0.002 & 0.002 & 0.003 \\
\hline Hubei & 0.002 & 0.002 & 0.003 & 0.003 & 0.002 & 0.003 & 0.003 & 0.003 \\
\hline Hunan & 0.002 & 0.002 & 0.002 & 0.002 & 0.002 & 0.002 & 0.002 & 0.002 \\
\hline Guangdong & 0.003 & 0.003 & 0.004 & 0.004 & 0.004 & 0.004 & 0.004 & 0.004 \\
\hline Guangxi & 0.003 & 0.003 & 0.004 & 0.004 & 0.004 & 0.004 & 0.004 & 0.004 \\
\hline Hainan & 0.006 & 0.006 & 0.006 & 0.005 & 0.006 & 0.006 & 0.007 & 0.008 \\
\hline Sichuan & 0.002 & 0.002 & 0.003 & 0.003 & 0.003 & 0.003 & 0.004 & 0.005 \\
\hline Guizhou & 0.004 & 0.004 & 0.004 & 0.004 & 0.004 & 0.004 & 0.004 & 0.005 \\
\hline Yunnan & 0.005 & 0.004 & 0.005 & 0.005 & 0.004 & 0.004 & 0.004 & 0.004 \\
\hline Shaanxi & 0.004 & 0.004 & 0.004 & 0.004 & 0.004 & 0.003 & 0.003 & 0.004 \\
\hline Gansu & 0.003 & 0.003 & 0.003 & 0.003 & 0.003 & 0.003 & 0.003 & 0.004 \\
\hline Qinghai & 0.003 & 0.002 & 0.002 & 0.002 & 0.002 & 0.002 & 0.002 & 0.002 \\
\hline Ningxia & 0.005 & 0.004 & 0.004 & 0.004 & 0.004 & 0.003 & 0.004 & 0.005 \\
\hline Xinjiang & 0.003 & 0.003 & 0.003 & 0.003 & 0.003 & 0.003 & 0.004 & 0.004 \\
\hline
\end{tabular}




\section{Continued}

\begin{tabular}{|c|c|c|c|c|c|c|c|}
\hline & 2008 & 2009 & 2010 & 2011 & 2012 & 2013 & 2014 \\
\hline Beijing & 0.012 & 0.013 & 0.013 & 0.016 & 0.016 & 0.018 & 0.019 \\
\hline Tianjin & 0.006 & 0.007 & 0.007 & 0.009 & 0.010 & 0.011 & 0.012 \\
\hline Hebei & 0.004 & 0.005 & 0.003 & 0.006 & 0.007 & 0.008 & 0.009 \\
\hline Shanxi & 0.005 & 0.006 & 0.005 & 0.005 & 0.006 & 0.008 & 0.009 \\
\hline Inner Mongolia & 0.008 & 0.007 & 0.003 & 0.008 & 0.009 & 0.011 & 0.012 \\
\hline Liaoning & 0.009 & 0.010 & 0.005 & 0.013 & 0.016 & 0.016 & 0.017 \\
\hline Jilin & 0.006 & 0.006 & 0.003 & 0.007 & 0.008 & 0.008 & 0.009 \\
\hline Heilongjiang & 0.005 & 0.006 & 0.006 & 0.008 & 0.009 & 0.009 & 0.010 \\
\hline Shanghai & 0.010 & 0.010 & 0.011 & 0.015 & 0.018 & 0.016 & 0.017 \\
\hline Jiangsu & 0.008 & 0.008 & 0.007 & 0.011 & 0.012 & 0.013 & 0.014 \\
\hline Zhejiang & 0.007 & 0.008 & 0.008 & 0.009 & 0.010 & 0.010 & 0.011 \\
\hline Anhui & 0.006 & 0.006 & 0.007 & 0.008 & 0.010 & 0.011 & 0.012 \\
\hline Fujian & 0.006 & 0.006 & 0.007 & 0.008 & 0.008 & 0.011 & 0.012 \\
\hline Jiangxi & 0.005 & 0.005 & 0.006 & 0.006 & 0.008 & 0.010 & 0.011 \\
\hline Shandong & 0.006 & 0.006 & 0.004 & 0.007 & 0.009 & 0.010 & 0.011 \\
\hline Henan & 0.004 & 0.005 & 0.005 & 0.006 & 0.007 & 0.007 & 0.008 \\
\hline Hubei & 0.004 & 0.005 & 0.004 & 0.006 & 0.007 & 0.009 & 0.010 \\
\hline Hunan & 0.003 & 0.003 & 0.004 & 0.005 & 0.006 & 0.006 & 0.007 \\
\hline Guangdong & 0.007 & 0.007 & 0.008 & 0.009 & 0.011 & 0.011 & 0.012 \\
\hline Guangxi & 0.005 & 0.005 & 0.005 & 0.006 & 0.007 & 0.008 & 0.009 \\
\hline Hainan & 0.011 & 0.014 & 0.017 & 0.022 & 0.023 & 0.026 & 0.027 \\
\hline Sichuan & 0.007 & 0.006 & 0.007 & 0.009 & 0.010 & 0.011 & 0.012 \\
\hline Guizhou & 0.006 & 0.006 & 0.007 & 0.007 & 0.007 & 0.009 & 0.010 \\
\hline Yunnan & 0.006 & 0.006 & 0.007 & 0.008 & 0.009 & 0.009 & 0.010 \\
\hline Shaanxi & 0.005 & 0.005 & 0.005 & 0.006 & 0.007 & 0.007 & 0.008 \\
\hline Gansu & 0.005 & 0.004 & 0.004 & 0.006 & 0.006 & 0.007 & 0.008 \\
\hline Qinghai & 0.003 & 0.004 & 0.003 & 0.003 & 0.004 & 0.005 & 0.006 \\
\hline Ningxia & 0.007 & 0.005 & 0.006 & 0.006 & 0.008 & 0.009 & 0.010 \\
\hline Xinjiang & 0.004 & 0.007 & 0.006 & 0.006 & 0.007 & 0.008 & 0.009 \\
\hline
\end{tabular}


Table 7. The value of economic development. Unit: yuan.

\begin{tabular}{|c|c|c|c|c|c|c|c|c|}
\hline & 2000 & 2001 & 2002 & 2003 & 2004 & 2005 & 2006 & 2007 \\
\hline Beijing & 24,127 & 26,980 & 30,730 & 34,777 & 40,916 & 45,993 & 50,467 & 60,096 \\
\hline Tianjin & 17353 & 19,141 & 21,387 & 25,544 & 30,575 & 35,783 & 41,163 & 47,970 \\
\hline Hebei & 7663 & 8362 & 9115 & 10251 & 12,487 & 14,814 & 16,962 & 20,033 \\
\hline Shanxi & 5722 & 6226 & 7082 & 8641 & 10,741 & 12,647 & 14,497 & 16,945 \\
\hline Inner Mongolia & 6502 & 7210 & 8146 & 10,015 & 12,728 & 16,371 & 20,693 & 26,521 \\
\hline Liaoning & 11,226 & 12,070 & 13,000 & 14,270 & 15,835 & 19,074 & 21,914 & 26,057 \\
\hline Jilin & 7351 & 7893 & 8714 & 9854 & 11,537 & 13,350 & 15,720 & 19,383 \\
\hline Heilongjiang & 8294 & 8900 & 9541 & 10,638 & 12,449 & 14,467 & 16,268 & 18,580 \\
\hline Shanghai & 30,047 & 31,799 & 35,445 & 38,486 & 46,338 & 52,535 & 58,837 & 62,041 \\
\hline Jiangsu & 11,773 & 12,925 & 14,397 & 16,830 & 20,031 & 24,953 & 28,943 & 34,294 \\
\hline Zhejiang & 13,461 & 14,713 & 16,978 & 20,149 & 24,784 & 27,703 & 31,874 & 37,411 \\
\hline Anhui & 4961 & 5313 & 5817 & 6375 & 7768 & 8810 & 10,055 & 12,045 \\
\hline Fujian & 11,194 & 11,691 & 12,739 & 14,125 & 16,235 & 18,646 & 21,471 & 25,908 \\
\hline Jiangxi & 4851 & 5221 & 5829 & 6678 & 8189 & 9440 & 11,145 & 13,322 \\
\hline Shandong & 9555 & 10,195 & 11,340 & 13,268 & 16,413 & 20,096 & 23,794 & 27,807 \\
\hline Henan & 5499 & 5959 & 6487 & 7376 & 9201 & 11,347 & 13,313 & 16,060 \\
\hline Hubei & 6293 & 6867 & 7437 & 8378 & 9898 & 11,554 & 13,360 & 16,386 \\
\hline Hunan & 5425 & 6120 & 6734 & 7589 & 9165 & 10,562 & 12,139 & 14,869 \\
\hline Guangdong & 12,736 & 13,852 & 15,365 & 17,798 & 20,876 & 24,647 & 28,747 & 33,890 \\
\hline Guangxi & 4652 & 4668 & 5099 & 5969 & 7461 & 8788 & 10,296 & 12,555 \\
\hline Hainan & 6894 & 7315 & 8041 & 8592 & 9812 & 11,165 & 12,810 & 14,923 \\
\hline Sichuan & 4956 & 5376 & 5890 & 6623 & 7895 & 9060 & 10,613 & 12,963 \\
\hline Guizhou & 2759 & 3000 & 3257 & 3701 & 4317 & 5119 & 6305 & 7878 \\
\hline Yunnan & 4770 & 5015 & 5366 & 5870 & 7012 & 7835 & 8970 & 10,609 \\
\hline Shaanxi & 4549 & 5511 & 6161 & 7057 & 8638 & 9899 & 12,840 & 15,546 \\
\hline Gansu & 4129 & 4386 & 4768 & 5429 & 6566 & 7477 & 8757 & 10,346 \\
\hline Qinghai & 5138 & 5774 & 6478 & 7346 & 8693 & 10,045 & 11,889 & 14,507 \\
\hline Ningxia & 5376 & 6039 & 6647 & 7734 & 9199 & 10,349 & 12,099 & 15,142 \\
\hline Xinjiang & 7470 & 7945 & 8457 & 9828 & 11,541 & 13,184 & 15,000 & 16,999 \\
\hline
\end{tabular}




\section{Continued}

\begin{tabular}{|c|c|c|c|c|c|c|c|}
\hline & 2008 & 2009 & 2010 & 2011 & 2012 & 2013 & 2014 \\
\hline Beijing & 64,491 & 66,940 & 73,856 & 81,658 & 87,475 & 93,213 & 95,344 \\
\hline Tianjin & 58,656 & 62,574 & 72,994 & 85,213 & 93,173 & 99,607 & 101,390 \\
\hline Hebei & 22,986 & 24,581 & 28,668 & 33,969 & 36,584 & 38,716 & 42,650 \\
\hline Shanxi & 21,506 & 21,522 & 26,283 & 31,357 & 33,628 & 34,813 & 39,221 \\
\hline Inner Mongolia & 34,869 & 39,735 & 47,347 & 57,974 & 63,886 & 67,498 & 70,692 \\
\hline Liaoning & 31,739 & 35,149 & 42,355 & 50,760 & 56,649 & 61,686 & 64,166 \\
\hline Jilin & 23,521 & 26,595 & 31,599 & 38,460 & 43,415 & 47,191 & 50,303 \\
\hline Heilongjiang & 21,740 & 22,447 & 27,076 & 32,819 & 35,711 & 37,509 & 41,610 \\
\hline Shanghai & 66,932 & 69,164 & 76,074 & 82,560 & 85,373 & 90,092 & 92,733 \\
\hline Jiangsu & 40,014 & 44,253 & 52,840 & 62,290 & 68,347 & 74,607 & 76,477 \\
\hline Zhejiang & 41,405 & 43,842 & 51,711 & 59,249 & 63,374 & 68,462 & 70,918 \\
\hline Anhui & 14,448 & 16,408 & 20,888 & 25,659 & 28,792 & 31,684 & 35,238 \\
\hline Fujian & 29,755 & 33,437 & 40,025 & 47,377 & 52,763 & 57,856 & 60,310 \\
\hline Jiangxi & 15,900 & 17,335 & 21,253 & 26,150 & 28,800 & 31,771 & 35,286 \\
\hline Shandong & 32,936 & 35,894 & 41,106 & 47,335 & 51,768 & 56,323 & 59,046 \\
\hline Henan & 19,181 & 20,597 & 24,446 & 28,661 & 31,499 & 34,174 & 37,837 \\
\hline Hubei & 19,858 & 22,677 & 27,906 & 34,197 & 38,572 & 42,613 & 45,592 \\
\hline Hunan & 18,147 & 20,428 & 24,719 & 29,880 & 33,480 & 36,763 & 40,122 \\
\hline Guangdong & 37,638 & 39,436 & 44,736 & 50,807 & 54,095 & 58,540 & 61,318 \\
\hline Guangxi & 14,652 & 16,045 & 20,219 & 25,326 & 27,952 & 30,588 & 34,270 \\
\hline Hainan & 17,691 & 19,254 & 23,831 & 28,898 & 32,377 & 35,317 & 38,847 \\
\hline Sichuan & 15,495 & 17,339 & 21,182 & 26,133 & 29,608 & 32,454 & 36,031 \\
\hline Guizhou & 9855 & 10,971 & 13,119 & 16,413 & 19,710 & 22,922 & 26,316 \\
\hline Yunnan & 12,570 & 13,539 & 15,752 & 19,265 & 22,195 & 25,083 & 28,639 \\
\hline Shaanxi & 19,700 & 21,947 & 27,133 & 33,464 & 38,564 & 42,692 & 45,628 \\
\hline Gansu & 12,421 & 13,269 & 16,113 & 19,595 & 21,978 & 24,296 & 28,137 \\
\hline Qinghai & 18,421 & 19,454 & 24,115 & 29,522 & 33,181 & 36,510 & 39,846 \\
\hline Ningxia & 19,609 & 21,777 & 26,860 & 33,043 & 36,394 & 39,420 & 42,907 \\
\hline Xinjiang & 19,797 & 19,942 & 25,034 & 30,087 & 33,796 & 37,847 & 40,822 \\
\hline
\end{tabular}


Table 8. The value of urbanization.

\begin{tabular}{|c|c|c|c|c|c|c|c|c|}
\hline & 2000 & 2001 & 2002 & 2003 & 2004 & 2005 & 2006 & 2007 \\
\hline Beijing & 0.79 & 0.78 & 0.79 & 0.79 & 0.80 & 0.84 & 0.84 & 0.85 \\
\hline Tianjin & 0.52 & 0.53 & 0.54 & 0.54 & 0.54 & 0.75 & 0.76 & 0.76 \\
\hline Hebei & 0.26 & 0.29 & 0.31 & 0.34 & 0.36 & 0.38 & 0.39 & 0.40 \\
\hline Shanxi & 0.36 & 0.35 & 0.38 & 0.39 & 0.40 & 0.42 & 0.43 & 0.44 \\
\hline Inner Mongolia & 0.43 & 0.43 & 0.44 & 0.45 & 0.46 & 0.47 & 0.49 & 0.50 \\
\hline Liaoning & 0.45 & 0.46 & 0.46 & 0.47 & 0.47 & 0.59 & 0.59 & 0.59 \\
\hline Jilin & 0.50 & 0.50 & 0.51 & 0.52 & 0.52 & 0.53 & 0.53 & 0.53 \\
\hline Heilongjiang & 0.52 & 0.52 & 0.53 & 0.53 & 0.53 & 0.53 & 0.54 & 0.54 \\
\hline Shanghai & 0.92 & 0.91 & 0.92 & 0.91 & 0.90 & 0.89 & 0.89 & 0.89 \\
\hline Jiangsu & 0.42 & 0.43 & 0.45 & 0.47 & 0.48 & 0.51 & 0.52 & 0.53 \\
\hline Zhejiang & 0.49 & 0.51 & 0.51 & 0.52 & 0.53 & 0.56 & 0.57 & 0.57 \\
\hline Anhui & 0.30 & 0.31 & 0.32 & 0.33 & 0.35 & 0.36 & 0.37 & 0.39 \\
\hline Fujian & 0.42 & 0.42 & 0.44 & 0.45 & 0.46 & 0.49 & 0.50 & 0.51 \\
\hline Jiangxi & 0.28 & 0.30 & 0.32 & 0.34 & 0.36 & 0.37 & 0.39 & 0.40 \\
\hline Shandong & 0.27 & 0.28 & 0.29 & 0.31 & 0.32 & 0.45 & 0.46 & 0.47 \\
\hline Henan & 0.23 & 0.24 & 0.26 & 0.27 & 0.29 & 0.31 & 0.32 & 0.34 \\
\hline Hubei & 0.40 & 0.41 & 0.41 & 0.42 & 0.43 & 0.43 & 0.44 & 0.44 \\
\hline Hunan & 0.30 & 0.31 & 0.32 & 0.33 & 0.35 & 0.37 & 0.39 & 0.40 \\
\hline Guangdong & 0.55 & 0.56 & 0.58 & 0.59 & 0.60 & 0.61 & 0.63 & 0.63 \\
\hline Guangxi & 0.27 & 0.27 & 0.29 & 0.29 & 0.32 & 0.34 & 0.35 & 0.36 \\
\hline Hainan & 0.40 & 0.41 & 0.42 & 0.43 & 0.44 & 0.45 & 0.46 & 0.47 \\
\hline Sichuan & 0.27 & 0.28 & 0.29 & 0.30 & 0.32 & 0.33 & 0.34 & 0.36 \\
\hline Guizhou & 0.24 & 0.24 & 0.24 & 0.25 & 0.26 & 0.27 & 0.27 & 0.28 \\
\hline Yunnan & 0.24 & 0.25 & 0.26 & 0.27 & 0.28 & 0.30 & 0.31 & 0.32 \\
\hline Shaanxi & 0.32 & 0.34 & 0.35 & 0.36 & 0.37 & 0.37 & 0.39 & 0.41 \\
\hline Gansu & 0.24 & 0.25 & 0.26 & 0.27 & 0.29 & 0.30 & 0.31 & 0.32 \\
\hline Qinghai & 0.35 & 0.36 & 0.38 & 0.38 & 0.38 & 0.39 & 0.39 & 0.40 \\
\hline Ningxia & 0.33 & 0.33 & 0.34 & 0.37 & 0.41 & 0.42 & 0.43 & 0.44 \\
\hline Xinjiang & 0.34 & 0.34 & 0.34 & 0.34 & 0.35 & 0.37 & 0.38 & 0.39 \\
\hline
\end{tabular}




\section{Continued}

\begin{tabular}{|c|c|c|c|c|c|c|c|}
\hline & 2008 & 2009 & 2010 & 2011 & 2012 & 2013 & 2014 \\
\hline Beijing & 0.85 & 0.85 & 0.86 & 0.86 & 0.86 & 0.86 & 0.91 \\
\hline Tianjin & 0.77 & 0.78 & 0.80 & 0.81 & 0.82 & 0.82 & 0.87 \\
\hline Hebei & 0.42 & 0.44 & 0.44 & 0.46 & 0.47 & 0.48 & 0.53 \\
\hline Shanxi & 0.45 & 0.46 & 0.48 & 0.50 & 0.51 & 0.53 & 0.58 \\
\hline Inner Mongolia & 0.52 & 0.53 & 0.56 & 0.57 & 0.58 & 0.59 & 0.64 \\
\hline Liaoning & 0.60 & 0.60 & 0.62 & 0.64 & 0.66 & 0.66 & 0.71 \\
\hline Jilin & 0.53 & 0.53 & 0.53 & 0.53 & 0.54 & 0.54 & 0.59 \\
\hline Heilongjiang & 0.55 & 0.56 & 0.56 & 0.57 & 0.57 & 0.57 & 0.62 \\
\hline Shanghai & 0.89 & 0.89 & 0.89 & 0.89 & 0.89 & 0.90 & 0.95 \\
\hline Jiangsu & 0.54 & 0.56 & 0.61 & 0.62 & 0.63 & 0.64 & 0.69 \\
\hline Zhejiang & 0.58 & 0.58 & 0.62 & 0.62 & 0.63 & 0.64 & 0.69 \\
\hline Anhui & 0.41 & 0.42 & 0.43 & 0.45 & 0.47 & 0.48 & 0.53 \\
\hline Fujian & 0.53 & 0.55 & 0.57 & 0.58 & 0.60 & 0.61 & 0.66 \\
\hline Jiangxi & 0.41 & 0.43 & 0.44 & 0.46 & 0.48 & 0.49 & 0.54 \\
\hline Shandong & 0.48 & 0.48 & 0.50 & 0.51 & 0.52 & 0.54 & 0.59 \\
\hline Henan & 0.36 & 0.38 & 0.39 & 0.41 & 0.42 & 0.44 & 0.49 \\
\hline Hubei & 0.45 & 0.46 & 0.50 & 0.52 & 0.54 & 0.55 & 0.60 \\
\hline Hunan & 0.42 & 0.43 & 0.43 & 0.45 & 0.47 & 0.48 & 0.53 \\
\hline Guangdong & 0.63 & 0.63 & 0.66 & 0.67 & 0.67 & 0.68 & 0.73 \\
\hline Guangxi & 0.38 & 0.39 & 0.40 & 0.42 & 0.44 & 0.45 & 0.50 \\
\hline Hainan & 0.48 & 0.49 & 0.50 & 0.51 & 0.52 & 0.53 & 0.58 \\
\hline Sichuan & 0.37 & 0.39 & 0.40 & 0.42 & 0.44 & 0.45 & 0.50 \\
\hline Guizhou & 0.29 & 0.30 & 0.34 & 0.35 & 0.36 & 0.38 & 0.43 \\
\hline Yunnan & 0.33 & 0.34 & 0.35 & 0.37 & 0.39 & 0.40 & 0.45 \\
\hline Shaanxi & 0.42 & 0.44 & 0.46 & 0.47 & 0.50 & 0.51 & 0.56 \\
\hline Gansu & 0.34 & 0.35 & 0.36 & 0.37 & 0.39 & 0.40 & 0.45 \\
\hline Qinghai & 0.41 & 0.42 & 0.45 & 0.46 & 0.47 & 0.49 & 0.54 \\
\hline Ningxia & 0.45 & 0.46 & 0.48 & 0.50 & 0.51 & 0.52 & 0.57 \\
\hline Xinjiang & 0.40 & 0.40 & 0.43 & 0.44 & 0.44 & 0.44 & 0.49 \\
\hline
\end{tabular}


Table 9. The value of fiscal spending.

\begin{tabular}{|c|c|c|c|c|c|c|c|c|}
\hline & 2000 & 2001 & 2002 & 2003 & 2004 & 2005 & 2006 & 2007 \\
\hline Beijing & 0.20 & 0.23 & 0.22 & 0.23 & 0.25 & 0.25 & 0.19 & 0.21 \\
\hline Tianjin & 0.13 & 0.14 & 0.14 & 0.15 & 0.15 & 0.15 & 0.15 & 0.16 \\
\hline Hebei & 0.09 & 0.10 & 0.10 & 0.11 & 0.11 & 0.11 & 0.12 & 0.13 \\
\hline Shanxi & 0.15 & 0.18 & 0.19 & 0.21 & 0.21 & 0.22 & 0.22 & 0.22 \\
\hline Inner Mongolia & 0.19 & 0.23 & 0.25 & 0.25 & 0.26 & 0.25 & 0.21 & 0.22 \\
\hline Liaoning & 0.12 & 0.14 & 0.14 & 0.15 & 0.16 & 0.18 & 0.18 & 0.19 \\
\hline Jilin & 0.16 & 0.18 & 0.18 & 0.18 & 0.20 & 0.21 & 0.20 & 0.21 \\
\hline Heilongjiang & 0.13 & 0.15 & 0.15 & 0.15 & 0.16 & 0.15 & 0.18 & 0.19 \\
\hline Shanghai & 0.15 & 0.16 & 0.17 & 0.20 & 0.22 & 0.22 & 0.20 & 0.21 \\
\hline Jiangsu & 0.08 & 0.09 & 0.09 & 0.10 & 0.11 & 0.11 & 0.11 & 0.12 \\
\hline Zhejiang & 0.08 & 0.10 & 0.11 & 0.12 & 0.11 & 0.11 & 0.11 & 0.11 \\
\hline Anhui & 0.11 & 0.13 & 0.14 & 0.14 & 0.15 & 0.15 & 0.17 & 0.20 \\
\hline Fujian & 0.09 & 0.10 & 0.09 & 0.10 & 0.10 & 0.10 & 0.11 & 0.12 \\
\hline Jiangxi & 0.12 & 0.14 & 0.16 & 0.16 & 0.16 & 0.16 & 0.17 & 0.19 \\
\hline Shandong & 0.08 & 0.09 & 0.09 & 0.10 & 0.10 & 0.09 & 0.10 & 0.10 \\
\hline Henan & 0.10 & 0.10 & 0.11 & 0.12 & 0.12 & 0.13 & 0.14 & 0.15 \\
\hline Hubei & 0.10 & 0.11 & 0.11 & 0.11 & 0.12 & 0.12 & 0.16 & 0.17 \\
\hline Hunan & 0.10 & 0.12 & 0.13 & 0.14 & 0.16 & 0.16 & 0.16 & 0.18 \\
\hline Guangdong & 0.13 & 0.14 & 0.14 & 0.14 & 0.14 & 0.14 & 0.11 & 0.12 \\
\hline Guangxi & 0.13 & 0.17 & 0.19 & 0.18 & 0.19 & 0.18 & 0.18 & 0.20 \\
\hline Hainan & 0.14 & 0.15 & 0.17 & 0.18 & 0.19 & 0.20 & 0.20 & 0.24 \\
\hline Sichuan & 0.12 & 0.15 & 0.16 & 0.16 & 0.17 & 0.17 & 0.19 & 0.21 \\
\hline Guizhou & 0.22 & 0.28 & 0.29 & 0.28 & 0.31 & 0.33 & 0.31 & 0.35 \\
\hline Yunnan & 0.22 & 0.25 & 0.25 & 0.26 & 0.27 & 0.26 & 0.26 & 0.29 \\
\hline Shaanxi & 0.18 & 0.21 & 0.22 & 0.20 & 0.22 & 0.22 & 0.22 & 0.23 \\
\hline Gansu & 0.20 & 0.24 & 0.26 & 0.26 & 0.27 & 0.28 & 0.27 & 0.30 \\
\hline Qinghai & 0.29 & 0.38 & 0.39 & 0.36 & 0.35 & 0.36 & 0.40 & 0.44 \\
\hline Ningxia & 0.25 & 0.35 & 0.38 & 0.32 & 0.32 & 0.35 & 0.32 & 0.34 \\
\hline Xinjiang & 0.16 & 0.19 & 0.24 & 0.23 & 0.22 & 0.24 & 0.26 & 0.26 \\
\hline
\end{tabular}




\section{Continued}

\begin{tabular}{|c|c|c|c|c|c|c|c|}
\hline & 2008 & 2009 & 2010 & 2011 & 2012 & 2013 & 2014 \\
\hline Beijing & 0.21 & 0.22 & 0.22 & 0.23 & 0.23 & 0.23 & 0.24 \\
\hline Tianjin & 0.17 & 0.18 & 0.18 & 0.19 & 0.19 & 0.20 & 0.21 \\
\hline Hebei & 0.14 & 0.15 & 0.16 & 0.17 & 0.17 & 0.17 & 0.18 \\
\hline Shanxi & 0.23 & 0.23 & 0.26 & 0.26 & 0.25 & 0.25 & 0.26 \\
\hline Inner Mongolia & 0.24 & 0.25 & 0.23 & 0.26 & 0.24 & 0.23 & 0.24 \\
\hline Liaoning & 0.20 & 0.20 & 0.21 & 0.21 & 0.21 & 0.21 & 0.22 \\
\hline Jilin & 0.22 & 0.23 & 0.25 & 0.25 & 0.23 & 0.23 & 0.24 \\
\hline Heilongjiang & 0.22 & 0.23 & 0.26 & 0.27 & 0.25 & 0.25 & 0.26 \\
\hline Shanghai & 0.21 & 0.22 & 0.22 & 0.23 & 0.22 & 0.22 & 0.23 \\
\hline Jiangsu & 0.13 & 0.13 & 0.14 & 0.15 & 0.14 & 0.14 & 0.15 \\
\hline Zhejiang & 0.12 & 0.12 & 0.14 & 0.14 & 0.13 & 0.14 & 0.15 \\
\hline Anhui & 0.22 & 0.24 & 0.26 & 0.27 & 0.26 & 0.25 & 0.26 \\
\hline Fujian & 0.12 & 0.13 & 0.14 & 0.15 & 0.15 & 0.16 & 0.17 \\
\hline Jiangxi & 0.22 & 0.24 & 0.25 & 0.27 & 0.26 & 0.27 & 0.28 \\
\hline Shandong & 0.10 & 0.11 & 0.12 & 0.13 & 0.13 & 0.13 & 0.14 \\
\hline Henan & 0.15 & 0.16 & 0.18 & 0.18 & 0.19 & 0.19 & 0.20 \\
\hline Hubei & 0.18 & 0.18 & 0.19 & 0.20 & 0.19 & 0.20 & 0.21 \\
\hline Hunan & 0.19 & 0.20 & 0.21 & 0.22 & 0.21 & 0.21 & 0.22 \\
\hline Guangdong & 0.12 & 0.12 & 0.14 & 0.15 & 0.14 & 0.15 & 0.16 \\
\hline Guangxi & 0.22 & 0.23 & 0.26 & 0.27 & 0.25 & 0.25 & 0.26 \\
\hline Hainan & 0.29 & 0.33 & 0.35 & 0.38 & 0.36 & 0.35 & 0.36 \\
\hline Sichuan & 0.27 & 0.28 & 0.29 & 0.29 & 0.27 & 0.26 & 0.27 \\
\hline Guizhou & 0.38 & 0.41 & 0.42 & 0.49 & 0.48 & 0.45 & 0.46 \\
\hline Yunnan & 0.31 & 0.34 & 0.37 & 0.41 & 0.40 & 0.40 & 0.41 \\
\hline Shaanxi & 0.26 & 0.27 & 0.27 & 0.29 & 0.27 & 0.25 & 0.26 \\
\hline Gansu & 0.36 & 0.39 & 0.43 & 0.43 & 0.41 & 0.41 & 0.42 \\
\hline Qinghai & 0.46 & 0.51 & 0.69 & 0.72 & 0.69 & 0.65 & 0.66 \\
\hline Ningxia & 0.37 & 0.39 & 0.41 & 0.42 & 0.41 & 0.39 & 0.40 \\
\hline Xinjiang & 0.30 & 0.32 & 0.40 & 0.42 & 0.41 & 0.41 & 0.42 \\
\hline
\end{tabular}


Table 10. The value of economic openness.

\begin{tabular}{|c|c|c|c|c|c|c|c|c|}
\hline & 2000 & 2001 & 2002 & 2003 & 2004 & 2005 & 2006 & 2007 \\
\hline Beijing & 1.89 & 1.72 & 1.53 & 1.77 & 2.14 & 2.40 & 0.82 & 0.79 \\
\hline Tianjin & 0.98 & 0.92 & 1.03 & 1.18 & 1.42 & 1.49 & 1.45 & 1.32 \\
\hline Hebei & 0.09 & 0.09 & 0.10 & 0.12 & 0.16 & 0.15 & 0.19 & 0.23 \\
\hline Shanxi & 0.10 & 0.10 & 0.11 & 0.13 & 0.18 & 0.15 & 0.18 & 0.25 \\
\hline Inner Mongolia & 0.17 & 0.12 & 0.13 & 0.13 & 0.14 & 0.15 & 0.13 & 0.14 \\
\hline Liaoning & 0.38 & 0.35 & 0.36 & 0.42 & 0.47 & 0.49 & 0.52 & 0.54 \\
\hline Jilin & 0.13 & 0.15 & 0.15 & 0.23 & 0.22 & 0.18 & 0.19 & 0.20 \\
\hline Heilongjiang & 0.09 & 0.09 & 0.10 & 0.11 & 0.13 & 0.15 & 0.20 & 0.23 \\
\hline Shanghai & 1.12 & 1.11 & 1.21 & 1.72 & 2.12 & 2.05 & 1.93 & 2.01 \\
\hline Jiangsu & 0.49 & 0.50 & 0.61 & 0.88 & 1.14 & 1.21 & 1.30 & 1.31 \\
\hline Zhejiang & 0.43 & 0.45 & 0.51 & 0.65 & 0.75 & 0.78 & 0.95 & 0.96 \\
\hline Anhui & 0.10 & 0.10 & 0.11 & 0.14 & 0.15 & 0.16 & 0.18 & 0.20 \\
\hline Fujian & 0.49 & 0.48 & 0.55 & 0.62 & 0.75 & 0.74 & 0.79 & 0.75 \\
\hline Jiangxi & 0.07 & 0.06 & 0.06 & 0.09 & 0.10 & 0.10 & 0.14 & 0.17 \\
\hline Shandong & 0.27 & 0.28 & 0.30 & 0.35 & 0.40 & 0.41 & 0.48 & 0.48 \\
\hline Henan & 0.04 & 0.04 & 0.05 & 0.06 & 0.08 & 0.07 & 0.08 & 0.09 \\
\hline Hubei & 0.07 & 0.07 & 0.07 & 0.09 & 0.10 & 0.12 & 0.15 & 0.15 \\
\hline Hunan & 0.06 & 0.06 & 0.06 & 0.07 & 0.10 & 0.09 & 0.10 & 0.10 \\
\hline Guangdong & 1.66 & 1.51 & 1.72 & 2.00 & 2.17 & 2.19 & 1.93 & 1.90 \\
\hline Guangxi & 0.09 & 0.07 & 0.09 & 0.11 & 0.13 & 0.13 & 0.15 & 0.16 \\
\hline Hainan & 0.23 & 0.28 & 0.28 & 0.32 & 0.42 & 0.27 & 0.30 & 0.52 \\
\hline Sichuan & 0.07 & 0.07 & 0.08 & 0.10 & 0.12 & 0.11 & 0.12 & 0.13 \\
\hline Guizhou & 0.06 & 0.05 & 0.05 & 0.07 & 0.09 & 0.07 & 0.09 & 0.11 \\
\hline Yunnan & 0.08 & 0.08 & 0.09 & 0.10 & 0.13 & 0.13 & 0.15 & 0.17 \\
\hline Shaanxi & 0.12 & 0.10 & 0.10 & 0.11 & 0.13 & 0.13 & 0.15 & 0.14 \\
\hline Gansu & 0.05 & 0.07 & 0.07 & 0.09 & 0.11 & 0.14 & 0.18 & 0.20 \\
\hline Qinghai & 0.06 & 0.06 & 0.05 & 0.08 & 0.12 & 0.07 & 0.14 & 0.08 \\
\hline Ningxia & 0.15 & 0.17 & 0.12 & 0.16 & 0.20 & 0.17 & 0.21 & 0.21 \\
\hline Xinjiang & 0.16 & 0.11 & 0.15 & 0.25 & 0.25 & 0.30 & 0.31 & 0.39 \\
\hline
\end{tabular}




\section{Continued}

\begin{tabular}{|c|c|c|c|c|c|c|c|}
\hline & 2008 & 2009 & 2010 & 2011 & 2012 & 2013 & 2014 \\
\hline Beijing & 2.02 & 1.40 & 1.68 & 1.78 & 1.58 & 1.49 & 1.54 \\
\hline Tianjin & 1.11 & 0.69 & 0.74 & 0.72 & 0.65 & 0.62 & 0.67 \\
\hline Hebei & 0.19 & 0.12 & 0.17 & 0.17 & 0.13 & 0.13 & 0.18 \\
\hline Shanxi & 0.17 & 0.08 & 0.12 & 0.10 & 0.08 & 0.08 & 0.13 \\
\hline Inner Mongolia & 0.10 & 0.06 & 0.06 & 0.07 & 0.05 & 0.05 & 0.10 \\
\hline Liaoning & 0.46 & 0.32 & 0.36 & 0.34 & 0.30 & 0.29 & 0.34 \\
\hline Jilin & 0.18 & 0.12 & 0.16 & 0.16 & 0.15 & 0.13 & 0.18 \\
\hline Heilongjiang & 0.23 & 0.13 & 0.20 & 0.24 & 0.19 & 0.18 & 0.23 \\
\hline Shanghai & 1.84 & 1.38 & 1.66 & 1.65 & 1.44 & 1.35 & 1.40 \\
\hline Jiangsu & 1.06 & 0.76 & 0.92 & 0.84 & 0.70 & 0.63 & 0.68 \\
\hline Zhejiang & 0.78 & 0.60 & 0.75 & 0.72 & 0.61 & 0.60 & 0.65 \\
\hline Anhui & 0.19 & 0.12 & 0.16 & 0.16 & 0.16 & 0.16 & 0.21 \\
\hline Fujian & 0.64 & 0.50 & 0.60 & 0.63 & 0.56 & 0.53 & 0.58 \\
\hline Jiangxi & 0.17 & 0.13 & 0.19 & 0.22 & 0.18 & 0.18 & 0.23 \\
\hline Shandong & 0.42 & 0.31 & 0.38 & 0.39 & 0.34 & 0.33 & 0.38 \\
\hline Henan & 0.08 & 0.05 & 0.06 & 0.09 & 0.12 & 0.13 & 0.18 \\
\hline Hubei & 0.16 & 0.10 & 0.14 & 0.14 & 0.10 & 0.10 & 0.15 \\
\hline Hunan & 0.09 & 0.06 & 0.08 & 0.08 & 0.07 & 0.07 & 0.12 \\
\hline Guangdong & 1.53 & 1.17 & 1.35 & 1.28 & 1.17 & 1.18 & 1.23 \\
\hline Guangxi & 0.15 & 0.14 & 0.15 & 0.16 & 0.16 & 0.16 & 0.21 \\
\hline Hainan & 0.26 & 0.23 & 0.35 & 0.40 & 0.36 & 0.32 & 0.37 \\
\hline Sichuan & 0.15 & 0.12 & 0.15 & 0.20 & 0.23 & 0.23 & 0.28 \\
\hline Guizhou & 0.09 & 0.05 & 0.05 & 0.07 & 0.07 & 0.07 & 0.12 \\
\hline Yunnan & 0.14 & 0.10 & 0.15 & 0.14 & 0.15 & 0.15 & 0.20 \\
\hline Shaanxi & 0.11 & 0.08 & 0.10 & 0.09 & 0.07 & 0.09 & 0.14 \\
\hline Gansu & 0.16 & 0.08 & 0.15 & 0.14 & 0.11 & 0.11 & 0.16 \\
\hline Qinghai & 0.06 & 0.04 & 0.05 & 0.04 & 0.04 & 0.05 & 0.10 \\
\hline Ningxia & 0.15 & 0.07 & 0.10 & 0.09 & 0.07 & 0.09 & 0.14 \\
\hline Xinjiang & 0.44 & 0.23 & 0.27 & 0.27 & 0.24 & 0.23 & 0.28 \\
\hline
\end{tabular}


Table 11. Variable descriptive statistical results.

\begin{tabular}{|c|c|c|c|c|c|c|}
\hline Variable & Mean & Median & Maximum & Minimum & Std. Dev. & Observations \\
\hline INE & 2.9805 & 2.8500 & 4.7600 & 1.8900 & 0.5999 & 435 \\
\hline $\mathrm{C}$ & 0.1618 & 0.1342 & 0.5316 & 0.0612 & 0.0907 & 435 \\
\hline $\mathrm{LC}$ & 0.0996 & 0.0842 & 0.3236 & 0.0442 & 0.0497 & 435 \\
\hline $\mathrm{SC}$ & 0.0381 & 0.0267 & 0.3137 & 0.0078 & 0.0427 & 435 \\
\hline $\mathrm{CC}$ & 0.0055 & 0.0044 & 0.0256 & 0.0016 & 0.0034 & 435 \\
\hline PGDP & 2.4027 & 1.8501 & 9.9607 & 0.2759 & 1.8937 & 435 \\
\hline UR & 0.4723 & 0.4466 & 0.9188 & 0.2320 & 0.1542 & 435 \\
\hline FI & 0.2148 & 0.1979 & 0.7164 & 0.0768 & 0.0994 & 435 \\
\hline OPEN & 0.3995 & 0.1583 & 2.3998 & 0.0413 & 0.5055 & 435 \\
\hline
\end{tabular}

The data source: China statistical yearbook, The Chinese tax yearbook.

model. When test results reject the null hypothesis, we choose the fixed effects regression model. Hausman test is used to select fixed effects regression model or random effects regression models. The null hypothesis choose random effects regression model. When test results reject the null hypothesis, we choose the fixed effects regression model.

We can see from Table 12, Likelihood ratio test and Hausman test results show that the P value of the model $1-3$ is far less than $1 \%$ under $1 \%$ significance level. We should reject the null hypothesis and choose fixed effects regression model. The results of Table 12 show regression results of model 1 - 3 by using fixed effects model.

As the results of Table 13 show, all variables get through $t$ test. Most variables reject the null hypothesis under $1 \%$ significance level in model 1 - 3. Part of variables rejects the null hypothesis under $5 \%$ or $10 \%$ significance level. R-squared and Adjusted R-squared of model 1 - 3 reach more than $85 \%$. It shows that the whole model has strong linear degree. $\mathrm{F}$ statistics of model 1 - 3 are significant under $1 \%$ significance level. At the same time, each variable symbol of model 1 - 3 is consistent with normal expectations. We can undertake economic analysis.

Model 1 is mainly used to demonstrate the relationship of Chinese actual situation and the "inverted U" hypothesis. According to the empirical results, the coefficient of per capita GDP is positive and the coefficient of per capita GDP square is negative. The test have good results. It shows that the relationship of income gap between urban and rural areas and economic development presents the Kuznets effect. With development of economy, income gap between urban and rural areas deteriorate in the beginning, and then improve.

In model 2, coefficient of tax contribution (C) is 3.38.The test has passed. It show that a positive relationship between income gap and tax contribution. If tax contribution increases $1 \%$, income gap between urban and rural areas will expand 3.38\%. General theory is that the relationship of tax contribution and income gap presents an opposite relationship, we can use tax to adjust residents' income gap, namely "high contribution -high fair, low contribution -low fair". Tax contribution is higher, it means national government has a stronger ability to concentrate financial resources and use resources. Then national government can provide more public goods to improve people's life and increase degree of economic and social justice. And low tax contribution is not beneficial to economic and social justice. Actual situation of other countries also can confirm this point of view. But the situation is different in our country. Since 2000, tax contribution of our country has been growing all the time, growth from $12.8 \%$ in 2000 to $19.6 \%$ in 2014. However, income gap between urban and rural areas have no decline, urban and rural income ratio rose from 2.76 in 2000 to 3.33 in 2009, down slightly after 2009, but still maintaining high proportion. At present, our country exist the objective fact of "high contribution-low fair". This is due to turnover tax contribution is the most important part in the tax contribution. In 2014, our country's tax contribution is $19.6 \%$. Turnover tax contribution is $10.1 \%$. Income tax contribution is just $5.0 \%$. Turnover tax contribution is more than $50 \%$ of total tax contribution, nearly twice as much as income tax contribution. High turnover tax contribution is not conducive to narrow income gap between urban and rural areas. In the "high contribution -high fair" country, income tax contribution is the most important part in the tax contribution. high tax contribution is conducive to narrow the income gap between urban and rural areas. 
Table 12. Likelihood ratio test and Hausman test.

\begin{tabular}{ccc}
\hline & Likelihood ratio test & Hausman test \\
\hline Model 1 & $105.3096^{* * *}$ & $56.1250^{* * *}$ \\
Model 2 & $63.8690^{* * *}$ & $192.1945^{* * *}$ \\
Model 3 & $63.8993^{* * *}$ & $186.2075^{* * *}$ \\
\hline
\end{tabular}

Note: $* * *, * *$ and $*$, is respectively at $1 \%, 5 \%$ and $10 \%$ significance levels.

Table 13. Regression results analysis.

\begin{tabular}{cccc}
\hline Variable & Model 1 & Model 2 & Model 3 \\
Constant & $2.9763^{* * *}(282.9252)$ & $1.9086^{* * *}(14.1188)$ & $2.4290^{* * *}(19.1040)$ \\
LOG(PGDP) & $0.1284^{* * *}(6.3165)$ & $0.1780^{* * *}(4.9300)$ & $0.1601^{* * *}(4.3665)$ \\
(LOG(PGDP))2 & $-0.0754^{* * *}(-6.1903)$ & $-0.1532^{* * *}(-11.1603)$ & $-0.1051^{* * *}(-6.5018)$ \\
C & & $3.3822^{* * *}(10.3095)$ & $5.0003^{* * *}(9.1589)$ \\
LC & & $1.6448^{* * *}(3.3063)$ \\
SC & & $-10.4574^{* *}(-2.3374)$ \\
CC & & $1.1518^{* * *}(5.3147)$ & $1.1392^{* * *}(4.7438)$ \\
UR & & $-2.4103^{* * *}(-8.9478)$ & $-2.4027^{* * *}(-8.5981)$ \\
FI & & $0.0688^{*}(1.6928)$ & $0.0781^{* *}(1.9769)$ \\
OPEN & 435 & 435 & 435 \\
Observation & 0.9149 & 0.9329 & 0.9367 \\
R-squared & 0.9081 & 0.9266 & 0.9305 \\
Adjusted R-squared & 134.4331 & 147.0548 & 151.6095 \\
F-statistic & 0.0000 & 0.0000 & 0.0000 \\
Prob (F-statistic) & & & \\
\hline
\end{tabular}

Note: $* * *, * *$ and $*$, is respectively at $1 \%, 5 \%$ and $10 \%$ significance levels.

Model 3 shows turnover tax contribution (LC), income tax contribution (SC) and property tax contribution (CC) on the influence of income gap between urban and rural areas. Coefficients of turnover tax contribution and income tax contribution are positive. They are 5.00 and 1.64 respectively. Coefficient of property tax contribution is negative, it is 10.46. This means that improvement of turnover tax contribution and income tax contribution will expand income gap between urban and rural areas; Improvement of property tax contribution will narrow the income gap between urban and rural areas. Coefficient of property tax contribution is very big. It indicates that property tax contribution has an obvious effect of narrowing income gap. Yet property tax income of our country is very small at present. Property tax can't play a role. The reason why income tax contribution expand income gap between urban and rural areas is that income tax system of our country is not perfect, such as adopting classified collection of individual income tax system, unreasonable expense deduction, unscientific tax rate structure, lack of tax collection and administration, etc.

Other control variables in model 2 and 3. Coefficient of urbanization (CZ) is positive. It will expand income gap between urban and rural areas and has a significant effect. There are two reasons: one is rich rural residents may transform into urban residents; the other one is economic results that create by rural migrant farmers mainly stay in the city, and urban residents enjoy most part of them. It leads to expand income gap between the urban and rural areas. Fiscal expenditure (FI) is helpful to narrow the income gap between urban and rural areas. This is because since 2002, Chinese fiscal spending pay more attention to the people's livelihood. The government 
increase spending on basic education, health care, social security, etc. Fiscal expenditure on rural residents is also increasing year by year. The government is gradually changing the fiscal policy of "urban bias". We can see from the empirical results, fiscal expenditure is indeed narrowing income gap between urban and rural areas. Economic openness (OPEN) has the function of expanding income gap between urban and rural areas. With Chinese economic developing, foreign trade focuses more on production technology and capital intensive products. Production technology requirement of these high-tech products is high. But the salary is also well. High technical level of workers is mainly concentrated in cities. Improvement of economic openness contributes to expand income gap between urban and rural areas.

\section{Conclusions and Policies}

We can draw the conclusion from above theoretical and empirical analysis: improvement of tax contribution is not conducive to narrow the income gap between urban and rural areas in our country. Improvement of turnover tax contribution and income tax contribution will expand income gap between urban and rural areas. Improvement of the property tax contribution will narrow income gap between urban and rural areas. Fiscal spending' increase will narrow the income gap between urban and rural areas. The urbanization' and economic openness' increase will expand the income gap between urban and rural areas. Based on the above analysis, we can put forward the following Suggestions:

We need to deepen our country's current structural tax cuts. "Structural tax cuts" means in order to achieve a specific goal, according to specific groups and specific tax, it reduce tax burden level. Since 2008, the structural tax cuts had played a role in adjusting income gap between urban and rural areas, but its role is limited. It failed to reduce macro tax burden and change tax structure. So we should continue to deepen reform of structural tax cuts. First of all, we should vigorously promote to replace the business tax with the value-added tax, reduce proportion of turnover tax, inhibit further increase of macro tax burden, and improve income level of resident department. The second, we should adjust current consumption tax system, expand consumption tax items, and adjust consumption tax rate. The third, we should improve personal income tax system, establish taxation pattern in accordance with Chinese national conditions, improve the system of expense deduction, and formulate scientific and reasonable tax rate. The fourth, we should improve the system of property tax, reform property tax system, and impose inheritance tax and gift tax.

It is very important for us to vigorously promote fiscal policies about people's livelihood. Compared with urban fiscal expenditure, financial expenditure in rural areas is still limited. Therefore, it is necessary to further increase proportion of fiscal expenditure and optimize structure of expenditure for supporting agriculture. In addition, it is also important to increase the rural education investment. We should ensure that rural education and urban education are equality. In order to realize urban and rural residents without differentiation, we need to improve the rural social security system.

In the process of urbanization, we should reform the household registration system, establish unified household registration system, and establish a social security network across the country. It is beneficial for urban and rural labor to realize barrier-free flow. At the same time, it also could realize urban and rural public service equalization.

In terms of economic openness, we should maintain a certain proportion of labor-intensive products. It is beneficial to ensure income level of rural unskilled labor. On the other hand, we should actively improve the skill levels of rural labor force to match production of capital-intensive products. That is a good way to ensure improvement of income level of rural residents, then narrow income gap between urban and rural areas.

\section{References}

[1] Deng, Z.J. (2001) Concentrating Financial Resources and Strengthening Fiscal Functions: The Core of President Jiang Zemin's Theory of Financing. Journal Xiamen University (Arts \& Social Sciences), 11, 5-9.

[2] Gao, P.Y. (2006) Establish Tax System about Adjusting the Gap Between Rich and Poor. Economy, 12, 50.

[3] Jia, K. (2008) Theory of Residents' Income Distribution-Based on Classification Stratification Adjustment of Rational Policy. Finance Research, 2, 2-5.

[4] Liu, H. (2012) Tax Structure and Income Inequality: Analysis based on World Bank WDI Data. Chinese Soft Science, 7, 179-185.

[5] Shi, Z.Y. (2014) The Effects of Standardized Tax Rates, Average Tax Rates, and the Distribution of Income on Per- 
sonal Income Tax Progressiveness. The Theory and Practice of Finance and Economics, 35, 70-74.

[6] Yue, X.M. (2014) Economic Development, Tax Adjustment and Chinese Urban Household Income Disparity. Journal of Hebei University of Economics and Business, 35, 66-72.

[7] Wan, Y. (2012) An Empirical Analysis of China's Income-Distribution Effects of Turnover Tax. Contemporary Finance \& Economics, 7, 21-30.

[8] Liu, Q.Z. (2011) Income Inequality and Redistribution of Individual Income Tax. Journal of Shanxi Finance and Economics University, 11, 1-9.

[9] Yi, L. and Nie, H.F. (2004) Analysis of Indirect Tax Burden's Influence on Income Distribution. Journal of Economic Studies, 5, 21-29.

[10] Hu, S.W. (2006) Taxation Adjustment on Structure, Equity and Harmony. Journal of Finance and Economy, 10, 6-11.

[11] Ouyang, H.S. and Xia, Y.F. (2011) Empirical Analysis of Personal Income Tax the Micro Tax Burden. Journal of Audit and Economic Studies, 26, 104-112.

[12] Chen, J.D. and Xia, Z.B. (2011) The Secondary Distribution Effect on the Regulation of Urban Residents Income Gap Analysis-Based on the Urban Household Survey Data in Anhui Province from 2007 to 2010. Journal of Economic Theory and Economic Management, 9, 87-92.

[13] The National Bureau of Statistics (2000-2014) China Statistical Yearbook. http://www.stats.gov.cn/tjsj/ndsj/

[14] The State Administration of Taxation (2000-2014) Chinese Tax Yearbook. http://tongji.cnki.net/Kns55/Navi/HomePage.aspx?id=N2015050184\&name=YZGRE\&floor=1 